amsppt.styFSp90a.tex

\title{
Pointwise compact and stable sets of measurable functions
}

\author{
S.SHELAH \& D.H.FREMLIN \\ Hebrew University, Jerusalem \\ University of Essex, Colchester, England
}

[University of Essex Mathematics Department Research Report 91-3]

Introduction In a series of papers culminating in [Ta84], M.Talagrand, the second author and others investigated at length the properties and structure of pointwise compact sets of measurable functions. A number of problems, interesting in themselves and important for the theory of Pettis integration, were solved subject to various special axioms. It was left unclear just how far the special axioms were necessary. In particular, several results depended on the fact that it is consistent to suppose that every countable relatively pointwise compact set of Lebesgue measurable functions is 'stable' in Talagrand's sense; the point being that stable sets are known to have a variety of properties not shared by all pointwise compact sets. In the present paper we present a model of set theory in which there is a countable relatively pointwise compact set of Lebesgue measurable functions which is not stable, and discuss the significance of this model in relation to the original questions. A feature of our model which may be of independent interest is the following: in it, there is a closed negligible set $Q \subseteq[0,1]^{2}$ such that whenever $D \subseteq[0,1]$ has outer measure 1 then

$$
Q^{-1}[D]=\{x: \exists y \in D,(x, y) \in Q\}
$$

has inner measure 1 (see $2 \mathrm{G}$ below).

1. The model We embark immediately on the central ideas of this paper, setting out a construction of a partially ordered set which forces a fairly technical proposition in measure theory (1S below); the relevance of this proposition to pointwise compact sets will be discussed in $\S 2$. The construction is complex, and rather than give it in a single stretch we develop it cumulatively in 1E, 1I, 1Q below; it is to be understood that each notation introduced in these paragraphs, as well as those in the definitions $1 \mathrm{~A}, 1 \mathrm{~K}, 1 \mathrm{~L}$, is to stand for the remainder of the section. After each part of the construction we give lemmas which can be dealt with in terms of the construction so far, even if their motivation is unlikely to be immediately clear.

When we come to results involving Forcing, we will try to follow the methods of [Ku80]; in particular, in a p.o.set, ' $p \leq q$ ' will always mean that $p$ is a stronger condition than $q$.

1A Definition If $\mathcal{A}$ is any family of sets not containing $\emptyset$, set

$$
\operatorname{dp}(\mathcal{A})=\min \{\#(I): I \cap A \neq \emptyset \forall A \in \mathcal{A}\} .
$$

Observe that $\operatorname{dp}(\mathcal{A})=0$ iff $\mathcal{A}=\emptyset$ and that $\operatorname{dp}(\mathcal{A} \cup \mathcal{B})$ is at most the cardinal sum of $\operatorname{dp}(\mathcal{A})$ and $\operatorname{dp}(\mathcal{B})$. (Of course much more can be said.)

1B Lemma Suppose that $n, l, k \in \mathbb{N}$, with $n, l$ not less than 2 , and that $\epsilon$ is such that $0<\epsilon \leq \frac{1}{2}$ and $l \epsilon^{k} \geq(k+2) \ln n$. Then there is a set $W \subseteq n \times n$ (we identify $n$ with the set of its predecessors) such that $\#(W) \leq \epsilon n^{2}$ and whenever $I \in[n]^{l}$ and $\bar{J}_{0}, \ldots, J_{l-1} \in[n]^{\leq k}$ are disjoint, there are $i \in I, j<l$ such that $\{i\} \times J_{j} \subseteq W$.

proof If $k=0$ this is trivial; suppose that $k>0$. Set $\Omega=\mathcal{P}(n \times n)$. Give $\Omega$ a probability for which the events $(i, j) \in W$, as $(i, j)$ runs over $n \times n$, are independent with probability $\epsilon$. If $W \in \Omega$ is a random set, then

$$
\operatorname{Pr}\left(\#(W) \leq \epsilon n^{2}\right)>\frac{1}{4}
$$

because $\epsilon \leq \frac{1}{2}$ and $\#(W)$ has the binomial distribution $B\left(n^{2}, \epsilon\right)$. On the other hand, if $J \in[n] \leq k$ and $i<n$, $\operatorname{Pr}(\{i\} \times J \subseteq W) \geq \epsilon^{k}$. So if $I \in[n]^{l}$ and $J_{0}, \ldots, J_{l-1}$ are disjoint members of $\left.[n]\right]^{\leq k}$, 


$$
\begin{aligned}
\operatorname{Pr}\left(\{i\} \times J_{j} \not \subset W \forall i \in I, j<l\right) & \leq\left(1-\epsilon^{k}\right)^{l^{2}} \\
& \leq \exp \left(-l^{2} \epsilon^{k}\right) .
\end{aligned}
$$

Accordingly

$$
\begin{aligned}
\operatorname{Pr}\left(\exists I \in[n]^{l},\right. \text { disjoint } & \left.J_{0}, \ldots, J_{l-1} \in[n]^{\leq k} \text { such that }\{i\} \times J_{j} \not \subset W \forall i \in I, j<l\right) \\
& \leq \#\left([n]^{l}\right) \#\left([n]^{\leq k}\right)^{l} \exp \left(-l^{2} \epsilon^{k}\right) \\
& \leq n^{l} n^{k l} \exp \left(-l^{2} \epsilon^{k}\right) \\
& =\exp \left((k+1) l \ln n-l^{2} \epsilon^{k}\right) \leq \frac{1}{4}
\end{aligned}
$$

because

$$
l^{2} \epsilon^{k}-(k+1) l \ln n \geq l \ln n \geq 2 \ln 2 .
$$

There must therefore be some $W \in \Omega$ of the type required.

Remark Compare the discussion of cliques in random graphs in [Sp87], pp. 18-20.

1C Lemma Let $m$ and $l$ be strictly positive integers and $\mathcal{A}$ a non-empty family of non-empty sets. Let $\mathbb{T}$ be the family of non-empty sets $\mathcal{T} \subseteq \mathcal{A}^{m}$. For $\mathcal{T} \in \mathbb{T}$ write $\mathcal{T}^{*}=\{\mathbf{t} \mid j: \mathbf{t} \in \mathcal{T}, j \leq m\} \subseteq \bigcup_{j \leq m} \mathcal{A}^{j}$. For $\mathcal{T}, \mathcal{T}_{0} \in \mathbb{T}$ say that $\mathcal{T} \preccurlyeq \mathcal{T}_{0}$ if $\mathcal{T} \subseteq \mathcal{T}_{0}$ and

$$
\operatorname{dp}\left(\left\{u: \mathbf{t}^{\frown} u \in \mathcal{T}^{*}\right\}\right) \geq \operatorname{dp}\left(\left\{u: \mathbf{t}^{\urcorner} u \in \mathcal{T}_{0}^{*}\right\}\right) / 2 l
$$

for every $\mathbf{t} \in \mathcal{T}^{*} \backslash \mathcal{T}$. Fix $\mathcal{T}_{0} \in \mathbb{T}$ and a cover $\left\langle\mathcal{S}_{i}\right\rangle_{i<2 l}$ of $\mathcal{T}_{0}$. Then there is a $\mathcal{T} \preccurlyeq \mathcal{T}_{0}$ such that $\mathcal{T} \subseteq \mathcal{S}_{i}$ for some $i<2 l$.

[Notation: In this context we use ordinary italics, ' $u$ ', for members of $\mathcal{A}$, and bold letters, ' $\mathbf{t}$ ', for finite sequences of members of $\mathcal{A}$.]

proof For $\mathbf{t} \in \mathcal{T}_{0}^{*} \backslash \mathcal{T}_{0}$ set

For $i<2 l$ define $\left\langle\mathcal{S}_{i}^{(j)}\right\rangle_{j \leq m}$ by setting $\mathcal{S}_{i}^{(m)}=\mathcal{S}_{i} \cap \mathcal{T}_{0}$,

$$
\alpha_{\mathbf{t}}=\operatorname{dp}\left(\left\{u: \mathbf{t}^{\frown} u \in \mathcal{T}_{0}^{*}\right\}\right) / 2 l>0 .
$$

$$
\mathcal{S}_{i}^{(j)}=\left\{\mathbf{t}: \mathbf{t} \in \mathcal{A}^{j} \cap \mathcal{T}_{0}^{*}, \operatorname{dp}\left(\left\{u: \mathbf{t}^{\frown} u \in \mathcal{S}_{i}^{(j+1)}\right\}\right) \geq \alpha_{\mathbf{t}}\right\}
$$

for $j<m$. An easy downwards induction (using the fact that dp is subadditive) shows that $\mathcal{T}_{0}^{*} \cap \mathcal{A}^{j}=$ $\bigcup_{i<2 l} \mathcal{S}_{i}^{(j)}$ for every $j \leq m$. In particular, there is some $i<2 l$ such that $\emptyset \in \mathcal{S}_{i}^{(0)}$. Now define $\mathcal{T}$ by and see that $\mathcal{T} \preccurlyeq \mathcal{T}_{0}$, as required.

$$
\left.\mathcal{T}=\left\{\mathbf{t}: \mathbf{t} \in \mathcal{A}^{m}, \mathbf{t}\right\rceil j \in \mathcal{S}_{i}^{(j)} \forall j \leq m\right\} \subseteq \mathcal{T}_{0} \cap \mathcal{S}_{i},
$$

1D Corollary Let $n, l, k$ and $W$ be as in Lemma 1B. Take $r \leq k$, let $Z$ be the cartesian product $n^{r}$ and set

$$
\tilde{W}=\{(i, z): i<n, z \in Z,(i, z(j)) \in W \forall j<r\} .
$$

Let $m, \mathcal{A}, \mathbb{T}$ and $\preccurlyeq$ be as in Lemma $1 \mathrm{C}$, and take $\mathcal{T}_{0} \in \mathbb{T}, H: \mathcal{T}_{0} \rightarrow n$ any function. Then

either there are $i<n, \mathcal{T} \preccurlyeq \mathcal{T}_{0}$ such that $H(\mathbf{t})=i$ for every $\mathbf{t} \in \mathcal{T}$

or there is a $J \in[n]^{\leq r l}$ such that for every $z \in(n \backslash J)^{r}$ there is a $\mathcal{T} \preccurlyeq \mathcal{T}_{0}$ such that $(H(\mathbf{t}), z) \in \tilde{W}$ for every $\mathbf{t} \in \mathcal{T}$.

\section{proof Set}

$$
A=\left\{z: z \in Z, \exists \mathcal{T} \preccurlyeq \mathcal{T}_{0} \text { such that }(H(\mathbf{t}), z) \in \tilde{W} \forall \mathbf{t} \in \mathcal{T}\right\} .
$$

If $A \supseteq(n \backslash J)^{r}$ for some $J \in[n] \leq r l$, we have the second alternative; suppose otherwise. Then we can find $z_{0}, \ldots, z_{l-1} \in Z \backslash A$ such that the sets $J_{j}=\left\{z_{j}(i): i<r\right\}$ are all disjoint. Each $J_{j}$ belongs to $[n] \leq k$, so by the choice of $W$,

$$
I=\left\{i:\{i\} \times J_{j} \not \subset W \forall j<l\right\}
$$

has cardinal less than $l$. Now observe that if $\mathbf{t} \in \mathcal{T}_{0}$ then either $H(\mathbf{t}) \in I$ or $\left(H(\mathbf{t}), z_{j}\right) \in \tilde{W}$ for some $j<l$. So we have a cover of $\mathcal{T}_{0}$ by the sets

$$
\begin{gathered}
\mathcal{S}_{i}=\{\mathbf{t}: H(\mathbf{t})=i\} \text { for } i \in I, \\
\mathcal{S}_{j}^{\prime}=\left\{\mathbf{t}:\left(H(\mathbf{t}), z_{j}\right) \in \tilde{W}\right\} \text { for } j<l .
\end{gathered}
$$


By Lemma $1 \mathrm{C}$, there is a $\mathcal{T} \preccurlyeq \mathcal{T}_{0}$ such that either $\mathcal{T} \subseteq \mathcal{S}_{i}$ for some $i \in I$ or $\mathcal{T} \subseteq \mathcal{S}_{j}^{\prime}$ for some $j<l$. But we cannot have $\mathcal{T} \subseteq \mathcal{S}_{j}^{\prime}$, because $z_{j} \notin A$; so $\mathcal{T} \subseteq \mathcal{S}_{i}$ for some $i$, and we have the first alternative.

Remark 1C-1D are of course elementary, but their significance is bound to be obscure; they will be used in $1 \mathrm{R}$ below. An essential feature of $1 \mathrm{C}$ is the fact that the denominator ' $2 l$ ' in the definition of $\preccurlyeq$ is independent of the size of $\mathcal{A}$.

1E Construction: part 1 (a) Take a sequence $\left\langle n_{k}\right\rangle_{k \in \mathbb{N}}$ of integers increasing so fast that

(i) $n_{0} \geq 4$;

(ii) $n_{k}>2^{k+1}$;

(iii) writing $\tilde{c}_{l}=\prod_{i<l} 2^{n_{i}}$, then

$\ln \left(2^{-k-1} n_{k}\right) \geq 2^{l}(k+1)\left(\tilde{c}_{l}^{l+1} \ln 2+\tilde{c}_{l}^{l-1} \ln n_{l-1}\right)$ for $1 \leq l \leq k ;$

(iv) $\ln n_{k} \geq\left(2^{k+1}\right)^{k}(k+2)$;

(v) writing $\lceil\alpha\rceil$ for the least integer greater than or equal to $\alpha$,

$(k+1) \ln \left(2\left\lceil\left(\ln n_{k}\right)^{2}\right\rceil\right) \leq 2^{-k}\left\lceil\ln \left(2^{-k-1} n_{k}\right)\right\rceil ;$

(vi) $2^{k} k\left\lceil\left(\ln n_{k}\right)^{2}\right\rceil\left(\prod_{i<k} 2^{n_{i}}\right)^{k+1} \leq n_{k}$;

(vii) $\ln \left(2^{-k-1} n_{k}\right) \geq(k+1) \ln \left(2 \tilde{c}_{k}^{k+1}+2 k\right)$

for every $k \in \mathbb{N}$.

For each $k \in \mathbb{N}$, let $V_{k}$ be the cartesian product $\prod_{i<k} n_{i}$.

(b) For each $k \in \mathbb{N}$, let $T_{k}$ be the set of those subsets $t$ of $V_{k}$ expressible as $t=\prod_{i<k} C_{i}(t)$ where $C_{i}(t) \subseteq n_{i}$ and $\#\left(C_{i}(t)\right) \geq\left(1-2^{-i-1}\right) n_{i}$ for each $i<k$. Set $T=\bigcup_{k \in \mathbb{N}} T_{k}$, and for $t \in T$ say that $\operatorname{rank}(t)=k$ if $t \in T_{k}$. For $t, t^{\prime} \in T$ say that $t \leq t^{\prime}$ if $\operatorname{rank}(t) \leq \operatorname{rank}\left(t^{\prime}\right)$ and $C_{i}(t)=C_{i}\left(t^{\prime}\right)$ for every $i<\operatorname{rank}(t)$. Then $T$ is a finitely-branching tree of height $\omega$ in which the $T_{k}$ are the levels and 'rank' is the rank function. For $t \in T$ write $T^{(t)}$ for the subtree $\left\{t^{\prime}: t^{\prime} \leq t\right.$ or $\left.t \leq t^{\prime}\right\}, \operatorname{suc}(t)$ for $\left\{t^{\prime}: t \leq t^{\prime}, \operatorname{rank}\left(t^{\prime}\right)=\operatorname{rank}(t)+1\right\}$.

(c) For $k \in \mathbb{N}$, set

$$
\gamma_{k}=(k+1) / \ln \left(\left\lceil 2^{-k-1} n_{k}\right\rceil\right)
$$

$2^{-k-1} n_{k}>1$ by (a)(ii) above. For $t \in T$ define $d_{t}: \mathcal{P} T \rightarrow \mathbb{R} \cup\{-\infty\}$ by writing

$$
d_{t}(S)=\gamma_{\operatorname{rank}(t)} \ln (\operatorname{dp}(\{C: t \times C \in S\}))
$$

for every $S \subseteq T$, allowing $d_{t}(S)=-\infty$ if $S \cap \operatorname{suc}(t)=\emptyset$. Observe that $d_{t}(T) \geq k+1$ whenever $\operatorname{rank}(t)=k$ (because

$$
\left.\operatorname{dp}\left(\left\{C: C \subseteq n_{k}, \#(C) \geq\left(1-2^{-k-1}\right) n_{k}\right\}\right) \geq\left\lceil 2^{-k-1} n_{k}\right\rceil .\right)
$$

(d) Let $\mathbb{Q}$ be the set of subtrees $q \subseteq T$ such that

$q \neq \emptyset$

if $t \leq t^{\prime} \in q$ then $t \in q$;

if $t \in q$ then $q \cap \operatorname{suc}(t) \neq \emptyset$;

writing $\delta_{k}(q)=\min \left\{d_{t}(q): t \in q \cap T_{k}\right\}$ for $k \in \mathbb{N}, \lim _{k \rightarrow \infty} \delta_{k}(q)=\infty$.

Observe that $\delta_{k}(T) \geq k+1$ for each $k \in \mathbb{N}$, so that $T \in \mathbb{Q}$ and $\mathbb{Q} \neq \emptyset$.

(e) For $q, q^{\prime} \in \mathbb{Q}$ say that $q \leq q^{\prime}$ if $q \subseteq q^{\prime}$. Then $(\mathbb{Q}, \leq, T)$ is a p.o.set (that is, a pre-ordered set with a top element, as in [Ku80]). Observe that if $t \in q \in \mathbb{Q}$ then $q \cap T^{(t)} \in \mathbb{Q}$ and $q \cap T^{(t)} \leq q$.

(f) For $q, q^{\prime} \in \mathbb{Q}$ and $k \in \mathbb{N}$ say that $q \leq_{k} q^{\prime}$ if $q \leq q^{\prime}$ and $q \cap T_{k}=q^{\prime} \cap T_{k}$ and

$$
d_{t}(q) \geq \min \left(k, d_{t}\left(q^{\prime}\right)\right)-2^{-k}
$$

for every $t \in q$. Note that $\leq_{k}$ is not transitive unless $k=0$.

Remarks Of course the point of the sequence $\left\langle n_{k}\right\rangle_{k \in \mathbb{N}}$ on which the rest of this construction will depend is that it increases 'as fast as we need it to'. The exact list given in (a) above is of no significance and will be used only as a list of clues to the (elementary) arguments below which depend on the rapidly-increasing nature of the sequence. This is why we have made no attempt to make the list as elegant or as short as possible.

Three elements may be distinguished within the construction of $\mathbb{Q}$. First, it is a p.o.set of rapidly branching trees; that is, if $t \in q \in \mathbb{Q}, q \cap \operatorname{suc}(t)$ is large compared with $T_{\operatorname{rank}(t)}$, except for $t$ of small rank. This is the basis of most of the (laborious but routine) work down to $1 \mathrm{P}$ below. Second, there is a natural $\mathbb{Q}$-name for a subset of $X=\prod_{k \in \mathbb{N}} n_{k}$ of large measure; a generic filter in $\mathbb{Q}$ leads to a branch of $T$ and 
hence to the $\Psi$ of $1 \mathrm{Q}(\mathrm{d})$. Third, the use of dp in the definition of 'rapidly branching' ((c)-(d) above) is what makes possible the side-step in the last part of the proof of $1 \mathrm{R}$.

1F Lemma $\mathbb{Q}$ is proper.

proof This is a special case of Proposition 1.18 in [Sh326]. (In fact, the arguments of 1G-1H below show that $\mathbb{Q}$ satisfies Axiom A, and is therefore proper; see [Ba84], 2.4.)

$1 \mathbf{G}$ Lemma Let $k \in \mathbb{N}$ and let $\zeta$ be an ordinal. Suppose that $A$ is a set with $\#(A) \leq \exp \left(2^{-k} / \gamma_{j}\right)-1$ for every $j \geq k$, and that $\tau$ is a $\mathbb{Q}$-name for a member of $A$. Let $\Delta$ be a $\mathbb{Q}$-name for a countable subset of $\zeta$. Then for every $q \in \mathbb{Q}$ there are a $q^{\prime} \leq_{k} q$, a function $H: T_{k} \rightarrow A$ and a countable (ground-model) set $D \subseteq \zeta$ such that

$$
\begin{gathered}
q^{\prime} \cap T^{(t)} \Vdash_{\mathbb{Q}} \tau=H(t) \forall t \in q^{\prime} \cap T_{k}, \\
q^{\prime} \Vdash_{\mathbb{Q}} \Delta \subseteq D .
\end{gathered}
$$

proof (a) Set $m=\#(A)$. The point is that if $j \geq k$ and $t \in T_{j}$ and $\left\langle S_{i}\right\rangle_{i \leq m}$ is a family of subsets of $T$, then

$$
\begin{aligned}
d_{t}\left(\bigcup_{i \leq m} S_{i}\right) & =\gamma_{j} \ln \left(\operatorname{dp}\left(\bigcup_{i \leq m}\left\{C: t \times C \in S_{i}\right\}\right)\right) \\
& \leq \gamma_{j} \ln \left(\sum_{i \leq m} \operatorname{dp}\left(\left\{C: t \times C \in S_{i}\right\}\right)\right) \\
& \leq \gamma_{j} \ln \left((m+1) \max _{i \leq m} \operatorname{dp}\left(\left\{C: t \times C \in S_{i}\right\}\right)\right) \\
& =\gamma_{j} \ln (m+1)+\max _{i \leq m} \gamma_{j} \ln \left(\operatorname{dp}\left(\left\{C: t \times C \in S_{i}\right\}\right)\right) \\
& \leq 2^{-k}+\max _{i \leq m} d_{t}\left(S_{i}\right) .
\end{aligned}
$$

(b) For each $a \in A$, let $S_{a}$ be the set

$$
\begin{aligned}
& \{t: t \in q, \operatorname{rank}(t) \geq k, \exists p \in \mathbb{Q}, D \in[\zeta] \leq \omega, \\
& \left.\quad p \leq_{k} q \cap T^{(t)}, p \Vdash_{\mathbb{Q}} \tau=a \& \Delta \subseteq D\right\} .
\end{aligned}
$$

If $t \in q \backslash S_{a}$ and $\operatorname{rank}(t) \geq k$, then $d_{t}\left(S_{a}\right)<\min \left(k, d_{t}(q)\right)-2^{-k}$. For if $S_{a} \cap \operatorname{suc}(t)=\emptyset, d_{t}\left(S_{a}\right)=-\infty$. While if $S_{a} \cap \operatorname{suc}(t) \neq \emptyset$, then for each $s \in \operatorname{suc}(t) \cap S_{a}$ we can find $p_{s} \in \mathbb{Q}$ and $D_{s} \in[\zeta] \leq \omega$ such that $p_{s} \leq_{k} q \cap T^{(s)}$, $p_{s} \Vdash_{\mathbb{Q}} \tau=a$ and $p_{s} \Vdash_{\mathbb{Q}} \Delta \subseteq D_{s}$. If we now set

$$
p=\bigcup_{s \in \operatorname{suc}(t) \cap S_{a}} p_{s}, \quad D=\bigcup_{s \in \operatorname{suc}(t) \cap S_{a}} D_{s},
$$

then $p \subseteq q \cap T^{(t)}$ and $p \Vdash_{\mathbb{Q}} \tau=a$ and $p \Vdash_{\mathbb{Q}} \Delta \subseteq D$. Because $t \notin S_{a}, p \underline{\Sigma}_{k} q \cap T^{(t)}$ and there must be an $s \in p$ such that $d_{s}(p)<\min \left(k, d_{s}\left(q \cap T^{(t)}\right)\right)-2^{-k}$; evidently $s=t$ and

as claimed.

$$
d_{t}\left(S_{a}\right)<\min \left(k, d_{t}(q)\right)-2^{-k},
$$

(c) Suppose, if possible, that there is a $t_{0} \in q \cap T_{k} \backslash \bigcup_{a \in A} S_{a}$. Set

$$
p=\left\{t: t \in q \cap T^{\left(t_{0}\right)}, t^{\prime} \notin \bigcup_{a \in A} S_{a} \forall t^{\prime} \leq t\right\} .
$$

Then $p$ is a subtree of $T$. For every $t \in p$ with $t \geq t_{0}$,

$$
d_{t}(q) \leq \max \left(\left\{d_{t}(p)\right\} \cup\left\{d_{t}\left(S_{a}\right): a \in A\right\}\right)+2^{-k}
$$

because $\#(A)=m$. But $d_{t}\left(S_{a}\right)<\bar{d}_{t}(q)-2^{-k}$ for every $a \in A$, by (b) above, so $d_{t}(p) \geq d_{t}(q)-2^{-k}$ (and $p \cap \operatorname{suc}(t) \neq \emptyset)$. This shows both that $p$ has no maximal elements and that $\delta_{i}(p) \geq \delta_{i}(q)-2^{-k}$ for every $i \geq k$, so that $p \in \mathbb{Q}$. Because $\mathbb{Q}$ is proper, we can find a $p^{\prime} \leq p$ and a countable $D \subseteq \zeta$ such that $p^{\prime} \Vdash \Delta \subseteq D$ ([Sh82], p. 81, III.1.16). Next, there are $p^{\prime \prime} \leq p^{\prime}, a \in A$ such that $p^{\prime \prime} \Vdash_{\mathbb{Q}} \tau=a$. Let $j \in \mathbb{N}$ be such that $\delta_{i}\left(p^{\prime \prime}\right) \geq k$ whenever $i \geq j$, and take $t \in p^{\prime \prime}$ such that $\operatorname{rank}(t) \geq \max (k, j)$. Then $p^{\prime \prime} \cap T^{(t)}$ witnesses that $t \in S_{a}$; which is impossible.

(d) Accordingly we have for every $t \in q \cap T_{k}$ an $H(t) \in A$, a countable set $D_{t}$ and a $p_{t} \in \mathbb{Q}$ such that

$$
\begin{gathered}
p_{t} \Vdash_{\mathbb{Q}} \tau=H(t) \& \Delta \subseteq D_{t}, \\
p_{t} \leq_{k} q \cap T^{(t)} .
\end{gathered}
$$


Set $q^{\prime}=\bigcup_{t \in q \cap T_{k}} p_{t}, D=\bigcup_{t \in q \cap T_{k}} D_{t}$; then $q^{\prime} \leq_{k} q, D$ is countable, $q^{\prime} \Vdash_{\mathbb{Q}} \Delta \subseteq D$ and $q^{\prime} \cap T^{(t)} \Vdash_{\mathbb{Q}} \tau=H(t)$ for every $t \in q \cap T_{k}$.

1H Lemma Let $\left\langle q_{k}\right\rangle_{k \in \mathbb{N}}$ be a sequence in $\mathbb{Q}$ such that $q_{2 k+2} \leq_{k+1} q_{2 k+1} \leq_{k} q_{2 k}$ for every $k \in \mathbb{N}$. Then $\hat{q}=\bigcap_{k \in \mathbb{N}} q_{k}$ belongs to $\mathbb{Q}$ and is accordingly a lower bound for $\left\{q_{k}: k \in \mathbb{N}\right\}$ in $\mathbb{Q}$; also $\hat{q} \cap T_{k+1}=q_{2 k+1} \cap T_{k+1}$ for each $k \in \mathbb{N}$.

proof Because each $q_{k}$ is a finitely-branching subtree of $T$ with no maximal elements, so is $\hat{q}$, and $d_{t}(\hat{q})=$ $\lim _{k \rightarrow \infty} d_{t}\left(q_{k}\right)$ for every $t \in \hat{q}$. Moreover, if $t \in \hat{q}$ and $k \leq l \in \mathbb{N}$,

$$
d_{t}\left(q_{2 l}\right) \geq \min \left(k, d_{t}\left(q_{2 k}\right)\right)-3.2^{-k}+3.2^{-l},
$$

(induce on $l$, using the definition of $\leq_{l}$ ), so we have

$$
\delta_{i}(\hat{q})=\lim _{l \rightarrow \infty} \delta_{i}\left(q_{2 l}\right) \geq \min \left(k, \delta_{i}\left(q_{2 k}\right)\right)-3.2^{-k}
$$

for every $i, k \in \mathbb{N}$; consequently $\lim _{i \rightarrow \infty} \delta_{i}(\hat{q})=\infty$ and $\hat{q} \in \mathbb{Q}$. Now if $k \in \mathbb{N}$ and $2 k+1 \leq l, q_{l+1} \cap T_{k+1}=$ $q_{l} \cap T_{k+1}$, so $\hat{q} \cap T_{k+1}=q_{2 k+1} \cap T_{k+1}$.

amsppt.styFSp90b.tex

Version of 16.9.92

1I Construction: part 2 Let $\kappa$ be the cardinal $\mathfrak{c}^{+}$(evaluated in the ground model).

(a) Let $\left(\left\langle\mathbb{P}_{\xi}\right\rangle_{\xi \leq \kappa},\left\langle\mathbb{Q}_{\xi}\right\rangle_{\xi<\kappa}\right)$ be a countable-support iteration of p.o.sets, as in [Ku80], chap. 8, such that each $\mathbb{Q}_{\xi}$ is a $\mathbb{P}_{\xi}$-name for a p.o.set with the same definition, interpreted in $V^{\mathbb{P}} \xi$, as the p.o.set $\mathbb{Q}$ of $1 \mathrm{E}$. (Note that $T$ is absolute, and so, in effect, is $\left\langle d_{t}\right\rangle_{t \in T}$, because each $d_{t}$ is determined by its values on the finite set $\mathcal{P}(\operatorname{suc}(t))$; so that the difference between $\mathbb{Q}$ and $\mathbb{Q}_{\xi}$ subsists in the power of $\mathbb{P}_{\xi}$ to add new subsets of $T$. Also each $\mathbb{Q}_{\xi}$ is 'full' in Kunen's sense.) Write $\mathbb{P}=\mathbb{P}_{\kappa}$.

(b) If $\zeta \leq \kappa, K \in[\zeta]^{<\omega}, k \in \mathbb{N}$ and $p, p^{\prime}$ belong to $\mathbb{P}_{\zeta}$, say that $p \leq_{K, k} p^{\prime}$ if $p \leq p^{\prime}$ and

$$
p\left\lceil\xi \Vdash_{\mathbb{P}_{\xi}} p(\xi) \leq_{k} p^{\prime}(\xi) \forall \xi \in K,\right.
$$

taking $\leq_{k}$ here to be a $\mathbb{P}_{\xi}$-name for the relation on $\mathbb{Q}_{\xi}$ corresponding to the relation $\leq_{k}$ on $\mathbb{Q}$ as defined in $1 \mathrm{E}(\mathrm{f})$. Of course $\leq_{K, k}$ is not transitive unless $K=\emptyset$ or $k=0$.

1J Lemma (a) $\mathbb{P}_{\zeta}$ is proper for every $\zeta \leq \kappa$.

(b) If $\xi<\kappa, \zeta \leq \kappa$ then $\mathbb{P}_{\xi+\zeta}$ may be identified with a dense subset of the iteration $\mathbb{P}_{\xi} * \mathbb{P}_{\zeta}^{\prime}$, where $\mathbb{P}_{\zeta}^{\prime}$ is a $\mathbb{P}_{\xi}$-name with the same definition, interpreted in $V^{\mathbb{P}_{\xi}}$, as the definition of $\mathbb{P}_{\zeta}$ in $V$.

(c) For every $\zeta<\kappa$,

$$
\mathbb{1}_{\mathbb{P}_{\zeta}} \mathbb{P}_{\mathbb{P}_{\zeta}} 2^{\omega}<\kappa .
$$

(d) If $\zeta \leq \kappa$ has uncountable cofinality, $A$ is a (ground-model) set, $\dot{f}$ is a $\mathbb{P}_{\zeta}$-name for a sequence in $A$

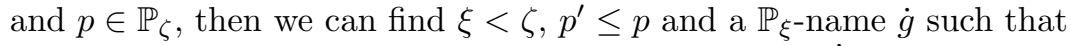

$$
p^{\prime} \vdash_{\mathbb{P}_{\zeta}} \dot{f}=\dot{g} .
$$

(e) If $A$ is a (ground-model) set and $\dot{f}$ is a $\mathbb{P}$-name for a sequence in $A$, then we can find a $\xi<\kappa$ and a $\mathbb{P}_{\xi}$-name $\dot{g}$ such that

$$
\mathbb{1}_{\mathbb{P}} \vdash_{\mathbb{P}} \dot{f}=\dot{g} .
$$

proof (a) This is just because $\mathbb{Q}$ is proper, as noted in 1F; see [Sh82], p. 90, Theorem III.3.2.

(b) This now follows by induction on $\zeta$. The inductive step to a successor ordinal is trivial, because if we can think of $\mathbb{P}_{\xi+\zeta}$ as dense in $\mathbb{P}_{\xi} * \mathbb{P}_{\zeta}^{\prime}$ then we can identify $\mathbb{Q}_{\xi+\zeta}$ with $\mathbb{Q}_{\zeta}^{\prime}$. As for the inductive step to limit $\zeta$, any member of $\mathbb{P}_{\xi+\zeta}$ can be regarded as $\left(p, p^{\prime}\right)$ where $p \in \mathbb{P}_{\xi}$ and $p^{\prime}$ is a $\mathbb{P}_{\xi}$-name for a member of $\mathbb{P}_{\zeta}^{\prime}$. On the other hand, given $\left(p, p^{\prime}\right) \in \mathbb{P}_{\xi} * \mathbb{P}_{\zeta}^{\prime}$, we have a $\mathbb{P}_{\xi}$-name $\dot{J}$ for the support of $p^{\prime}$ which in $V^{\mathbb{P}_{\xi}}$ is a countable subset of $\zeta$. But because $\mathbb{P}_{\xi}$ is proper there are a $p_{1} \leq p$ and a countable ground-model set $I \subseteq \zeta$ such that $p_{1} \Vdash_{\mathbb{P}_{\xi}} \dot{J} \subseteq I$ ([Sh82], p. 81, III.1.16). Now $\left(p_{1}, p^{\prime}\right)$ can be re-interpreted as a member of $\mathbb{P}_{\xi+\zeta}$ stronger than $\left(p, p^{\prime}\right)$. Thus $\mathbb{P}_{\xi+\zeta}$ is dense in $\mathbb{P}_{\xi} * \mathbb{P}_{\zeta}^{\prime}$, as claimed.

(c) [Sh82], p. 96, III.4.1.

(d) [Sh82], p. 171, V.4.4.

(e) By [Sh82], p. 96, III.4.1, $\mathbb{P}$ satisfies the $\kappa$-c.c.; because $\kappa$ is regular, (d) gices the result.

1K Definition Let $\zeta \leq \kappa, p \in \mathbb{P}_{\zeta}$. 
(a) Define $\mathbf{U}(p),\left\langle p^{(\mathbf{u})}\right\rangle_{\mathbf{u} \in \mathbf{U}(p)}$ as follows. A finite function $\mathbf{u} \subseteq \zeta \times T$ belongs to $\mathbf{U}(p)$ if either $\mathbf{u}=\emptyset$, in which case $p^{(\mathbf{u})}=p$,

or $\mathbf{u}=\mathbf{v} \cup\{(\xi, t)\}$ where $\mathbf{v} \in \mathbf{U}(p), \operatorname{dom}(\mathbf{v}) \subseteq \xi<\zeta$, and

$$
p^{(\mathbf{v})} \mid \xi \Vdash_{\mathbb{P}_{\xi}} t \in p^{(\mathbf{v})}(\xi)
$$

in which case $p^{(\mathbf{u})}$ is defined by writing

$$
\begin{gathered}
p^{(\mathbf{u})}(\eta)=p^{(\mathbf{v})}(\eta) \forall \eta \in \zeta \backslash\{\xi\}, \\
p^{(\mathbf{u})}(\xi)=p^{(\mathbf{v})}(\xi) \cap T^{(t)} .
\end{gathered}
$$

(b) Observe that if $\mathbf{u} \in \mathbf{U}(p)$ then $p^{(\mathbf{u})}(\xi)=p(\xi)$ for $\xi \in \zeta \backslash \operatorname{dom}(\mathbf{u}), p^{(\mathbf{u})}(\xi)=p(\xi) \cap T^{(\mathbf{u}(\xi))}$ if $\xi \in \operatorname{dom}(\mathbf{u})$; $\mathbf{U}(p)$ is just the set of finite functions $\mathbf{u}$ for which these formulae define such a $p^{(\mathbf{u})} \in \mathbb{P}_{\zeta}$. Of course $p^{(\mathbf{u})} \leq p$ for every $\mathbf{u} \in \mathbf{U}(p)$.

(c) Note that if $\xi \leq \zeta, p \in \mathbb{P}_{\zeta}, \mathbf{u} \in \mathbf{U}(p)$ then $\mathbf{u} \mid \xi \in \mathbf{U}\left(p\lceil\xi)\right.$ and $\left(p\lceil\xi)^{(\mathbf{u}\lceil\xi)}=p^{(\mathbf{u})} \mid \xi\right.$.

(d) If $p \in \mathbb{P}_{\zeta}, \mathbf{u} \in \mathbf{U}(p)$ and $\mathbf{v} \subseteq \zeta \times T$ is a finite function such that $\operatorname{dom}(\mathbf{u}) \subseteq \operatorname{dom}(\mathbf{v})$ and $\mathbf{u}(\xi) \leq \mathbf{v}(\xi)$ in $T$ for every $\xi \in \operatorname{dom}(\mathbf{u})$, then $\mathbf{v} \in \mathbf{U}(p)$ iff $\mathbf{v} \in \mathbf{U}\left(p^{(\mathbf{u})}\right)$, and in this case $p^{(\mathbf{v})}=\left(p^{(\mathbf{u})}\right)^{(\mathbf{v})}$ (induce on \# $\#(\mathbf{v})$ ).

(e) We shall mostly be using not the whole of $\mathbf{U}(p)$ but the sets $\mathbf{U}(p ; K, k)=\mathbf{U}(p) \cap T_{k}^{K}$ for $K \in[\zeta]^{<\omega}$, $k \in \mathbb{N}$, writing $T_{k}^{K}$ for the set of functions from $K$ to $T_{k}$.

1L Definition For $\zeta \leq \kappa, K \in[\zeta]^{<\omega}, k \in \mathbb{N}$ and $p \in \mathbb{P}_{\zeta}$, say that $p$ is $(K, k)$-fixed if for every $\eta \in K$, $\mathbf{u} \in \mathbf{U}(p ; K \cap \eta, k)$ there is a (ground-model) set $A \subseteq T_{k}$ such that

$$
p^{(\mathbf{u})}\left\lceil\eta \Vdash_{\mathbb{P}_{\eta}} p(\eta) \cap T_{k}=A\right. \text {. }
$$

Equivalently, $p$ is $(K, k)$-fixed if $\mathbf{U}(p ; K, k) \supseteq \mathbf{U}\left(p_{1} ; K, k\right)$ for every $p_{1} \leq p$.

1M Lemma Suppose $\zeta \leq \kappa, K \in[\zeta]^{<\omega}, k \geq 1$ and that $A$ is a finite set with $2^{c^{m}} a^{c^{m-1}} \leq \exp \left(2^{-k} / \gamma_{i}\right)$

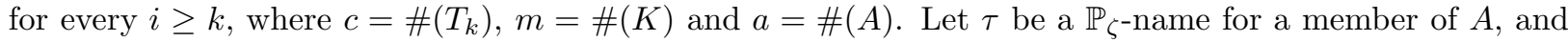
$\Delta$ a $\mathbb{P}_{\zeta}$-name for a countable subset of $\kappa$. Then for any $p \in \mathbb{P}_{\zeta}$ there are $p_{1} \leq_{K, k} p$, a function $H: T_{k}^{K} \rightarrow A$ and a countable (ground-model) set $D \subseteq \kappa$ such that

$$
\begin{aligned}
& p_{1} \text { is }(K, k) \text {-fixed, } \\
& p_{1}^{(\mathbf{u})} \Vdash_{\mathbb{P}_{\zeta}} \tau=H(\mathbf{u}) \forall \mathbf{u} \in \mathbf{U}\left(p_{1} ; K, k\right) \text {, } \\
& p_{1} \Vdash_{\mathbb{P}_{\zeta}} \Delta \subseteq D \text {. }
\end{aligned}
$$

proof Induce on $m=\#(K)$. If $m=0$ we may take any $a \in A, p_{1}^{\prime} \leq p$ such that $p_{1}^{\prime} \Vdash \tau=a$, and (again using [Sh82], III.1.16, this time based on 1Ja) a countable $D$ and a $p_{1} \leq p_{1}^{\prime}$ such that $p_{1} \Vdash_{\mathbb{P}_{\zeta}} \Delta \subseteq D$; now set $H(\emptyset)=a$.

For the inductive step to $\#(K)=m \geq 1$, let $\xi$ be $\max K$. As explained in $1 \mathrm{Jb}, \mathbb{P}_{\zeta}$ may be regarded as a dense subset of $\mathbb{P}_{\xi+1} * \mathbb{P}^{\prime}$; arguing momentarily in $V^{\mathbb{P}_{\xi+1}}$ we can find a $\mathbb{P}_{\xi+1}$-name $\hat{r}_{0}$ for a member of $\mathbb{P}^{\prime}$, a $\mathbb{P}_{\xi+1}$-name $\tau^{\prime}$ for a member of $A$ and a $\mathbb{P}_{\xi+1}$-name $\Delta^{\prime}$ for a countable set such that

$$
\begin{gathered}
\left(p\left\lceil\xi+1, \hat{r}_{0}\right) \leq p \text { in } \mathbb{P}_{\xi+1} * \mathbb{P}^{\prime},\right. \\
\left(p\left\lceil\xi+1, \hat{r}_{0}\right) \Vdash_{\mathbb{P}_{\xi+1} * \mathbb{P}^{\prime}} \tau^{\prime}=\tau,\right. \\
\left(p\left\lceil\xi+1, \hat{r}_{0}\right) \Vdash_{\mathbb{P}_{\xi+1} * \mathbb{P}^{\prime}} \Delta \subseteq \Delta^{\prime} .\right.
\end{gathered}
$$

Now let $\Delta_{0}^{\prime}$ be a $\mathbb{P}_{\xi+1}$-name for a countable subset of $\zeta \backslash(\xi+1)$ such that

$$
\mathbb{1}_{\mathbb{P}_{\xi+1}} \Vdash_{\mathbb{P}_{\xi+1}} \operatorname{supp}\left(\hat{r}_{0}\right)=\Delta_{0}^{\prime} .
$$

Because $\#(A)=a<2^{c^{m}} a^{c^{m-1}} \leq \exp \left(2^{-k} / \gamma_{i}\right)$ for every $i \geq k$, we can use Lemma $1 \mathrm{G}$ in $V^{\mathbb{P}_{\xi}}$ to find $\tilde{H}, \tilde{q}$, $\tilde{\Delta}$ such that

$\tilde{H}$ is a $\mathbb{P}_{\xi}$-name for a function from $T_{k}$ to $A$,

$\tilde{\Delta}$ is a $\mathbb{P}_{\xi}$-name for a countable subset of $\kappa$,

$$
\begin{gathered}
\tilde{q} \in \mathbb{Q}_{\xi}, \\
p \nmid \xi \Vdash_{\mathbb{P}_{\xi}} \tilde{q} \leq k p(\xi), \\
p \nmid \xi \Vdash_{\mathbb{P}_{\xi}}\left(\tilde{q} \cap T^{(t)} \Vdash_{\mathbb{Q}_{\xi}} \tau^{\prime}=\tilde{H}(t) \forall t \in \tilde{q} \cap T_{k}\right), \\
p\left\lceil\xi \Vdash_{\mathbb{P}_{\xi}}\left(\tilde{q} \Vdash_{\mathbb{Q}_{\xi}} \Delta^{\prime} \cup \Delta_{0}^{\prime} \subseteq \tilde{\Delta}\right) .\right.
\end{gathered}
$$

Now consider the pair $\left(\tilde{H}, \tilde{q} \cap T_{k}\right)$. This can be regarded as a $\mathbb{P}_{\xi}$-name for a member of $A_{1}=A^{T_{k}} \times \mathcal{P} T_{k}$, and $a_{1}=\#\left(A_{1}\right)=2^{c} a^{c}$, so

$$
2^{c^{m-1}} a_{1}^{c^{m-2}}=2^{2 c^{m-1}} a^{c^{m-1}} \leq 2^{c^{m}} a^{c^{m-1}} \leq \exp \left(2^{-k} / \gamma_{j}\right) \forall i \geq k .
$$


The inductive hypothesis therefore tells us that there are $\hat{p}_{1} \leq_{K \cap \xi, k} p\left\lceil\xi, H^{*}: T_{k}^{K \cap \xi} \rightarrow A^{T_{k}}, F^{*}: T_{k}^{K \cap \xi} \rightarrow\right.$ $\mathcal{P} T_{k}$ and a countable $D \subseteq \kappa$ such that

for every $\mathbf{u} \in \mathbf{U}\left(\hat{p}_{1} ; K \cap \xi, k\right)$, and

$$
\begin{gathered}
\hat{p}_{1} \text { is }(K \cap \xi, k) \text {-fixed, } \\
\hat{p}_{1}^{(\mathbf{u})} \Vdash_{\mathbb{P}_{\xi}} \tilde{H}=H^{*}(\mathbf{u}) \& \tilde{q} \cap T_{k}=F^{*}(\mathbf{u})
\end{gathered}
$$

At this point we observe that

$$
\hat{p}_{1} \Vdash_{\mathbb{P}_{\xi}} \tilde{\Delta} \subseteq D .
$$

$$
\hat{p}_{1} \Vdash_{\mathbb{P}_{\xi}}\left(\tilde{q} \Vdash_{\mathbb{Q}_{\xi}} \operatorname{supp}\left(\hat{r}_{0}\right) \subseteq D\right) .
$$

Now the only difference between $\mathbb{P}_{\xi+1} * \mathbb{P}^{\prime}$ and $\mathbb{P}_{\zeta}$ is that for members of the former their supports have to be regarded as $\mathbb{P}_{\xi+1}$-names for countable subsets of $\zeta$, and these are not always reducible to countable ground-model sets. But in the present case this difficulty does not arise and we have a $p_{1} \in \mathbb{P}_{\zeta}$ defined by saying that $p_{1}\left|\xi=\hat{p}_{1}, p_{1}\right| \xi \Vdash_{\mathbb{P}_{\xi}} p_{1}(\xi)=\tilde{q}$, and $p_{1}\left\lceil\eta \Vdash_{\mathbb{P}_{\eta}} p_{1}(\eta)=\hat{r}_{0}(\eta)\right.$ for $\xi<\eta<\zeta$; then $\operatorname{supp}\left(p_{1}\right) \subseteq$ $\operatorname{supp}\left(\hat{p}_{1}\right) \cup\{\xi\} \cup(D \cap \zeta)$ is countable.

Consequently $p_{1} \in \mathbb{P}_{\zeta}$ is well-defined and now, setting $H\left(\mathbf{u}^{\frown} t\right)=H^{*}(\mathbf{u})(t)$ for $\mathbf{u} \in T_{k}^{K \cap \xi}, t \in T_{k}$,

and finally

$$
\begin{gathered}
p_{1} \leq_{K, k} p, \\
p_{1} \Vdash_{\mathbb{P}} \Delta \subseteq D, \\
\mathbf{U}\left(p_{1} ; K, k\right)=\left\{\mathbf{u} \uparrow t: \mathbf{u} \in \mathbf{U}\left(\hat{p}_{1} ; K \cap \xi, k\right), t \in F^{*}(\mathbf{u})\right\}, \\
p_{1}^{(\mathbf{v})} \Vdash_{\mathbb{P}_{\zeta}} \tau=H(\mathbf{v}) \forall \mathbf{v} \in \mathbf{U}\left(p_{1} ; K, k\right)
\end{gathered}
$$

so that $p_{1}$ is $(K, k)$-fixed, and the induction proceeds.

$1 \mathbf{N}$ Lemma Suppose $\zeta \leq \kappa,\left\langle K_{k}\right\rangle_{k \in \mathbb{N}}$ is an increasing sequence of finite subsets of $\zeta,\left\langle p_{k}\right\rangle_{k \in \mathbb{N}}$ is a sequence in $\mathbb{P}_{\zeta}$; suppose that

$$
p_{2 k+2} \leq_{K_{k}, k+1} p_{2 k+1} \leq_{K_{k}, k} p_{2 k}
$$

for every $k \in \mathbb{N}$ and that $\bigcup_{k \in \mathbb{N}} \operatorname{supp}\left(p_{k}\right) \subseteq \bigcup_{k \in \mathbb{N}} K_{k}$. Then there is a $\hat{p} \in \mathbb{P}_{\zeta}$ such that $\hat{p} \leq p_{k}$ for every $k \in \mathbb{N}, \operatorname{supp}(\hat{p}) \subseteq \bigcup_{k \in \mathbb{N}} K_{k}$ and

so that

$$
\begin{gathered}
\hat{p} \mid \xi \Vdash_{\mathbb{P}_{\xi}} \hat{p}(\xi) \cap T_{k}=p_{2 k+1} \cap T_{k} \quad \forall k \in \mathbb{N}, \xi \in K_{k}, \\
\hat{p} \mid \xi \Vdash_{\mathbb{P}_{\xi}} \hat{p}(\xi) \cap T_{k+1}=p_{2 k+2} \cap T_{k+1} \quad \forall k \in \mathbb{N}, \xi \in K_{k+1},
\end{gathered}
$$

for every $k \in \mathbb{N}$.

$$
\mathbf{U}\left(\hat{p} ; K_{k}, k\right) \supseteq \mathbf{U}\left(p_{2 k+1} ; K_{k}, k\right) \text { and } \mathbf{U}\left(\hat{p} ; K_{k}, k+1\right) \supseteq \mathbf{U}\left(\hat{p}_{k+2} ; K_{k}, k+1\right)
$$

proof For each $\xi<\zeta$ choose $\hat{p}(\xi)$ such that

$$
\mathbb{1}_{\mathbb{P}_{\xi}} \Vdash_{\mathbb{P}_{\xi}} \hat{p}(\xi)=\bigcap_{k \in \mathbb{N}} p_{k}(\xi) .
$$

An easy induction on $\xi$ shows that $\hat{p} \mid \xi \in \mathbb{P}_{\xi}$ for every $\xi \leq \zeta$; for if $\xi \in \zeta \backslash \bigcup_{k \in \mathbb{N}} K_{k}$ then

while if $k \in \mathbb{N}$ and $\xi \in K_{k}$ then

$$
\mathbb{1}_{\mathbb{P}_{\xi}} \Vdash_{\mathbb{P}_{\xi}} \hat{p}(\xi)=T=\mathbb{1}_{\mathbb{Q}_{\xi}}
$$

so that by Lemma $1 \mathrm{H}$,

$$
\hat{p} \mid \xi \Vdash_{\mathbb{P}_{\xi}} p_{2 l+2}(\xi) \leq_{l+1} p_{2 l+1}(\xi) \leq_{l} p_{2 l}(\xi) \forall l \geq k,
$$

$$
\begin{gathered}
\hat{p} \mid \xi \Vdash_{\mathbb{P}_{\xi}} \hat{p}(\xi) \in \mathbb{Q}_{\xi} \& \hat{p}(\xi) \cap T_{k+1}=p_{2 k+1} \cap T_{k+1}=p_{2 k+2} \cap T_{k+1} \\
\& \hat{p}(\xi) \cap T_{k}=p_{2 k+1} \cap T_{k} .
\end{gathered}
$$

It follows at once that $\mathbf{U}\left(\hat{p} ; K_{k}, k\right) \supseteq \mathbf{U}\left(p_{2 k+1} ; K_{k}, k\right), \mathbf{U}\left(\hat{p} ; K_{k}, k+1\right) \supseteq \mathbf{U}\left(p_{2 k+2} ; K_{k}, k+1\right)$ for every $k \in \mathbb{N}$.

10 Lemma Suppose that $0<\zeta \leq \kappa, \sigma$ is a $\mathbb{P}_{\zeta}$-name for a member of $\prod_{k \in \mathbb{N}} n_{k}$, and $p \in \mathbb{P}_{\zeta}$. Then we can find a $\hat{p}$ and sequences $\left\langle K_{k}\right\rangle_{k \in \mathbb{N}},\left\langle H_{k}\right\rangle_{k \in \mathbb{N}}$ such that

$\hat{p} \in \mathbb{P}_{\zeta}, \hat{p} \leq p ;$

$\left\langle K_{k}\right\rangle_{k \in \mathbb{N}}$ is an increasing sequence of subsets of $\zeta$, \#( $\left.K_{k}\right) \leq k+1$ for every $k, K_{0}=\{0\}$;

$\operatorname{supp}(\hat{p}) \subseteq \bigcup_{k \in \mathbb{N}} K_{k}$

$\hat{p}$ is $\left(K_{k}, k\right)$-fixed and $\left(K_{k}, k+1\right)$-fixed for every $k$;

$H_{k}$ is a function from $T_{k+1}^{K_{k}}$ to $n_{k}$ for every $k$;

$\hat{p}^{(\mathbf{u})} \Vdash_{\mathbb{P}_{\zeta}} \sigma(k)=H_{k}(\mathbf{u})$ whenever $k \in \mathbb{N}$ and $\mathbf{u} \in \mathbf{U}\left(\hat{p} ; K_{k}, k+1\right)$.

proof Using Lemma $1 \mathrm{M}$, we can find sequences $\left\langle p_{k}\right\rangle_{k \in \mathbb{N}},\left\langle K_{k}\right\rangle_{k \in \mathbb{N}}$ and $\left\langle H_{k}\right\rangle_{k \in \mathbb{N}}$ such that 


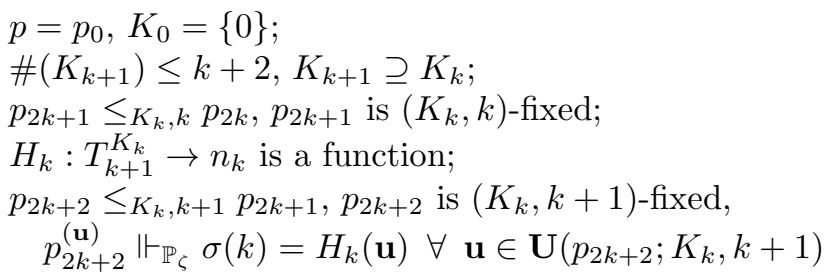

for every $k \in \mathbb{N}$. Furthermore, we may do this in such a way that $\bigcup_{k \in \mathbb{N}} K_{k}$ includes $\bigcup_{k \in \mathbb{N}} \operatorname{supp}\left(p_{k}\right)$. We need of course to know that the $n_{k}$ are rapidly increasing; specifically, that

(when choosing $p_{2 k+1}$ ) and that

$$
2^{c_{k}^{k+1}} \leq \exp \left(2^{-k} / \gamma_{i}\right) \forall i \geq k
$$

$$
2^{c_{k+1}^{k+1}} n_{k}^{c_{k+1}^{k}} \leq \exp \left(2^{-k-1} / \gamma_{i}\right) \forall i \geq k+1
$$

(when choosing $p_{2 k+2}$ ), where we write $c_{k}=\#\left(T_{k}\right)$. But as $c_{k} \leq \prod_{i<k} 2^{n_{i}}$, this is a consequence of $1 \mathrm{E}(\mathrm{a})(\mathrm{i})$ and (iii).

Armed with the sequences $\left\langle p_{k}\right\rangle_{k \in \mathbb{N}},\left\langle K_{k}\right\rangle_{k \in \mathbb{N}}$ we may now use Lemma $1 \mathrm{~N}$ to find a $\hat{p}$ as described there. Because $p_{2 k+1}$ is $\left(K_{k}, k\right)$-fixed and $\mathbf{U}\left(\hat{p} ; K_{k}, k\right) \supseteq \mathbf{U}\left(p_{2 k+1} ; K_{k}, k\right)$ we must have equality here and $\hat{p}$ is $\left(K_{k}, k\right)$-fixed for every $k \in \mathbb{N}$. Similarly, $\hat{p}$ is $\left(K_{k}, k+1\right)$-fixed for every $k$. Moreover, if $\mathbf{u} \in \mathbf{U}\left(\hat{p} ; K_{k}, k+1\right)=$ $\mathbf{U}\left(p_{2 k+2} ; K_{k}, k+1\right)$ we have $\hat{p}^{(\mathbf{u})} \leq p_{2 k+2}^{(\mathbf{u})}$, so

as required.

$$
\hat{p}^{(\mathbf{u})} \Vdash_{\mathbb{P}_{\zeta}} \sigma(k)=H_{k}(\mathbf{u})
$$

$1 \mathbf{P}$ Lemma Suppose that $\zeta \leq \kappa, p \in \mathbb{P}_{\zeta}, k \in \mathbb{N}, K \in[\zeta]^{<\omega}$ and $\mathbf{V}$ is a non-empty subset of $\mathbf{U}(p ; K, k)$. Then we have a $p_{1}=\bigvee_{\mathbf{v} \in \mathbf{V}} p^{(\mathbf{v})}$ defined (up to $\leq$-equivalence in $\mathbb{P}_{\zeta}$ ) by saying

if $\xi \in \zeta \backslash K$ then $p_{1}(\xi)=p(\xi)$;

if $\xi \in K$ then

for $\mathbf{u} \in\{\mathbf{v} \mid \xi: \mathbf{v} \in \mathbf{V}\}$.

$$
\left(p_{1}\lceil\xi)^{(\mathbf{u})} \Vdash_{\mathbb{P}_{\xi}} p_{1}(\xi)=\bigcup\left\{p(\xi) \cap T^{(t)}: \exists \mathbf{v} \in \mathbf{V} \text { such that } \mathbf{v}\left\lceil\xi+1=\mathbf{u}^{\frown} t\right\}\right.\right.
$$

Now $p_{1} \leq p$ and if $\xi<\zeta, t \in p_{1}(\xi), \operatorname{rank}(t) \geq k$ we shall have

so if $\xi<\zeta, i \geq k$ we have

$$
p_{1}\left\lceil\xi \Vdash_{\mathbb{P}_{\xi}} \operatorname{suc}(t) \cap p_{1}(\xi)=\operatorname{suc}(t) \cap p(\xi) ;\right.
$$

$$
p_{1} \mid \xi \Vdash_{\mathbb{P}_{\xi}} \delta_{i}\left(p_{1}(\xi)\right) \geq \delta_{i}(p(\xi)) .
$$

If $p_{2} \leq p_{1}$ there is some $\mathbf{v} \in \mathbf{V}$ such that $p_{2}$ is compatible with $p_{1}^{(\mathbf{v})}=p^{(\mathbf{v})}$. If $k \leq l \in \mathbb{N}, K \subseteq L \in[\zeta]<\omega$ then

$$
\mathbf{U}\left(p_{1} ; L, l\right)=\{\mathbf{w}: \mathbf{w} \in \mathbf{U}(p ; L, l), \exists \mathbf{v} \in \mathbf{V} \text { such that } \mathbf{v}(\xi) \leq \mathbf{w}(\xi) \forall \xi \in K\},
$$

and $p_{1}^{(\mathbf{w})}=p^{(\mathbf{w})}$ for every $\mathbf{w} \in \mathbf{U}\left(p_{1} ; L, l\right)$; consequently, $p_{1}$ is $(L, l)$-fixed if $p$ is.

proof Requires only a careful reading of the definitions.

Remark Note that $1 \mathrm{G}-1 \mathrm{P}$ are based just on the fact that $\mathbb{Q}$ is a p.o.set of rapidly branching trees; the exact definition of 'rapidly branching' in $1 \mathrm{E}(\mathrm{c})$ is relevant only to some of the detailed calculations. Similar ideas may be found in [BJSp89] and [Sh326].

1Q Construction: part 3 (a) Set $X=\prod_{k \in \mathbb{N}} n_{k}$. Then $X$, with its product topology, is a compact metric space. Let $\mu$ be the natural Radon probability on $X$, the product of the uniform probabilities on the factors.

(b) For each $k \in \mathbb{N}$ set $l_{k}=\left\lceil\left(\ln n_{k}\right)^{2}\right\rceil$. Take $W_{k}^{\prime} \subseteq n_{k} \times n_{k}$ such that $\#\left(W_{k}^{\prime}\right) \leq 2^{-k-1} n_{k}^{2}$ and whenever $I \in\left[n_{k}\right]^{l_{k}}$ and $J_{0}, \ldots, J_{l_{k}-1}$ are disjoint members of $\left[n_{k}\right] \leq k$, there are $i \in I$ and $j<l_{k}$ such that $\{i\} \times J_{j} \subseteq$ $W_{k}^{\prime}$. (This is possible by Lemma $1 \mathrm{~B}$ and $1 \mathrm{E}(\mathrm{a})(\mathrm{iv})$.) Set $W_{k}=W_{k}^{\prime} \cup\left\{(i, i): i<n_{k}\right\}$.

Write $R$ for

$$
\left\{(x, y): x, y \in X,(x(k), y(k)) \in W_{k} \forall k \in \mathbb{N},\{k: x(k)=y(k)\} \text { is finite }\right\}
$$

then $R$ is negligible for the product measure of $X \times X$. For $r \in \mathbb{N}$ write $R_{r}$ for the set

$$
\left\{\left(x,\left\langle y_{i}\right\rangle_{i<r}\right): x \in X,\left(x, y_{i}\right) \in R \forall i<r\right\} \subseteq X \times X^{r} .
$$

We shall frequently wish to interpret the formulae for the sets $X, R_{r}$ in $V^{\mathbb{P}}$; when doing so we will write $\ulcorner X\urcorner,\left\ulcorner R_{r}\right\urcorner$.

(c) Write 
$\mathcal{L}=\left\{\left\langle L_{k}\right\rangle_{k \in \mathbb{N}}: L_{k} \subseteq n_{k} \forall k \in \mathbb{N}, \prod_{k \in \mathbb{N}} \#\left(L_{k}\right) / n_{k}>0\right\}$.

Again, we shall wish to distinguish between the ground-model set $\mathcal{L}$ and a corresponding $\mathbb{P}$-name $\ulcorner\mathcal{L}\urcorner$.

(d) For each $k \in \mathbb{N}$ let $\Phi_{k}$ be the $\mathbb{P}$-name for a subset of $n_{k}$ defined (up to equivalence) by saying that $p \vdash_{\mathbb{P}} \Phi_{k}=C_{k}(t)$

whenever $\operatorname{rank}(t)>k$ and $p(0) \subseteq T^{(t)}$. (Here $C_{k}(t)$ is the $k$ th factor of $t$, as described in $1 \mathrm{E}(\mathrm{b})$.) Let $\Psi_{k}, \Psi$ be $\mathbb{P}$-names for the subsets of $\ulcorner X\urcorner$ given by

Then we have

$$
\mathbb{1}_{\mathbb{P}} \Vdash_{\mathbb{P}} \Psi_{k}=\left\{\sigma: \sigma \in\ulcorner X\urcorner, \sigma(i) \in \Phi_{i} \forall i \geq k\right\}, \Psi=\bigcup_{k \in \mathbb{N}} \Psi_{k} .
$$

so that

$$
\mathbb{1}_{\mathbb{P}} \Vdash_{\mathbb{P}} \#\left(\Phi_{k}\right) \geq\left(1-2^{-k-1}\right) n_{k} \forall k \in \mathbb{N},
$$

$$
\mathbb{1}_{\mathbb{P}} \Vdash_{\mathbb{P}}\ulcorner\mu\urcorner(\Psi)=1 .
$$

1R Main Lemma If $r \in \mathbb{N}$ and $D \subseteq X^{r}$ is a (ground-model) set such that $D \cap\left(\prod_{k \in \mathbb{N}} L_{k}\right)^{r} \neq \emptyset$ for every (ground-model) sequence $\left\langle L_{k}\right\rangle_{k \in \mathbb{N}} \in \mathcal{L}$, then for every (ground-model) sequence $\left\langle L_{k}\right\rangle_{k \in \mathbb{N}} \in \mathcal{L}$

$$
\mathbb{1}_{\mathbb{P}} \Vdash_{\mathbb{P}} \Psi \cap \prod_{k \in \mathbb{N}} L_{k} \subseteq\left\ulcorner R_{r}\right\urcorner^{-1}\left[D \cap\left(\prod_{k \in \mathbb{N}} L_{k}\right)^{r}\right] .
$$

proof (a) Let $\left\langle L_{k}\right\rangle_{k \in \mathbb{N}} \in \mathcal{L}$, let $\sigma$ be a $\mathbb{P}$-name such that

$$
\mathbb{1}_{\mathbb{P}} \Vdash_{\mathbb{P}} \sigma \in \Psi \cap \prod_{k \in \mathbb{N}} L_{k}
$$

and let $p \in \mathbb{P}$. Write $D^{\prime}$ for $D \cap\left(\prod_{k \in \mathbb{N}} L_{k}\right)^{r}$. Let $k_{0} \geq r, p_{1} \leq p$ be such that $p_{1} \Vdash_{\mathbb{P}} \sigma \in \Psi_{k_{0}}$. By Lemma $1 \mathrm{O}$, we have a $p_{2} \leq p_{1}$, an increasing sequence $\left\langle K_{k}\right\rangle_{k \in \mathbb{N}}$ of finite subsets of $\kappa$, and a sequence $\left\langle H_{k}\right\rangle_{k \in \mathbb{N}}$ of functions such that

$p_{2}$ is $\left(K_{k}, k\right)$-fixed and $\left(K_{k}, k+1\right)$-fixed for every $k \in \mathbb{N}$,

$p_{2}^{(\mathbf{u})} \Vdash_{\mathbb{P}} \sigma(k)=H_{k}(\mathbf{u})$ whenever $\mathbf{u} \in \mathbf{U}\left(p_{2} ; K_{k}, k+1\right), k \in \mathbb{N}$;

$\bigcup_{k \in \mathbb{N}} K_{k} \supseteq \operatorname{supp}\left(p_{2}\right)$;

$0 \in K_{0}, \#\left(K_{k}\right) \leq k+1$ for every $k \in \mathbb{N}$.

(b) For $k \geq k_{0}$, let $Z_{k}$ be the cartesian product set $n_{k}^{r}$ and take $\tilde{W}_{k}$ to be $\left\{(i, z): i<n_{k}, z \in Z_{k},(i, z(j)) \in W_{k} \forall j<r\right\}$.

Set $\mathcal{A}_{k}=\mathcal{P} n_{k} \backslash\{\emptyset\}$ and $\mathbb{T}_{k}=\mathcal{P}\left(\mathcal{A}_{k}^{K_{k}}\right) \backslash\{\emptyset\}$; define $\preccurlyeq_{k}$ on $\mathbb{T}_{k}$ as in Lemma 1C, taking $l_{k}$ and $K_{k}$ (with the order induced by that of $\kappa)$ in place of $l$ and $m$ there.

For each $\mathbf{u} \in \mathbf{U}\left(p_{2} ; K_{k}, k\right)$ set

$$
\mathcal{T}_{\mathbf{u}}=\left\{\mathbf{c}: \mathbf{u}^{\wedge} \mathbf{c} \in \mathbf{U}\left(p_{2} ; K_{k}, k+1\right)\right\} \in \mathbb{T}_{k},
$$

where for $\mathbf{u} \in T_{k}^{K}, \mathbf{c} \in\left(\mathcal{P} n_{k}\right)^{K}$ we write

$$
\mathbf{u}^{\wedge} \mathbf{c}=\langle\mathbf{u}(\xi) \times \mathbf{c}(\xi)\rangle_{\xi \in K} .
$$

By Corollary 1D, we may find for each such $\mathbf{u}$ a $w_{\mathbf{u}}<n_{k}$ and a set $J_{\mathbf{u}} \subseteq n_{k}$ such that $\#\left(J_{\mathbf{u}}\right) \leq r l_{k}$ and either there is a $\mathcal{T} \preccurlyeq{ }_{k} \mathcal{T}_{\mathbf{u}}$ such that $H_{k}\left(\mathbf{u}^{\wedge} \mathbf{c}\right)=w_{\mathbf{u}}$ for every $\mathbf{c} \in \mathcal{T}$

or for every $z \in\left(n_{k} \backslash J_{\mathbf{u}}\right)^{r}$ there is a $\mathcal{T} \preccurlyeq_{k} \mathcal{T}_{\mathbf{u}}$ such that $\left(H_{k}\left(\mathbf{u}^{\wedge} \mathbf{c}\right), z\right) \in \tilde{W}_{k}$, that is,

for every $\mathbf{c} \in \mathcal{T}$.

$$
\left(H_{k}\left(\mathbf{u}^{\wedge} \mathbf{c}\right), z(j)\right) \in W_{k} \forall j<r,
$$

Set

so that

$$
\begin{gathered}
\tilde{I}_{k}=\left\{w_{\mathbf{u}}: \mathbf{u} \in \mathbf{U}\left(p_{2} ; K_{k}, k\right)\right\}, \\
\tilde{J}_{k}=\bigcup\left\{J_{\mathbf{u}}: \mathbf{u} \in \mathbf{U}\left(p_{2} ; K_{k}, k\right)\right\},
\end{gathered}
$$

by $1 \mathrm{E}(\mathrm{a})(\mathrm{vi})$.

$$
\begin{gathered}
\#\left(\tilde{I}_{k}\right) \leq \#\left(\mathbf{U}\left(p_{2} ; K_{k}, k\right)\right) \leq \#\left(T_{k}^{K_{k}}\right) \leq\left(\prod_{i<k} 2^{n_{i}}\right)^{k+1}, \\
\#\left(\tilde{J}_{k}\right) \leq k l_{k}\left(\prod_{i<k} 2^{n_{i}}\right)^{k+1} \leq 2^{-k} n_{k}
\end{gathered}
$$

(c) Let $k_{1} \geq \max \left(k_{0}, 1\right)$ be such that

$$
\delta_{k}\left(p_{2}(0)\right) \geq 2, \tilde{J}_{k} \nsupseteq L_{k}
$$

for every $k \geq k_{1}$. Take any $\mathbf{v}^{*} \in \mathbf{U}\left(p_{2} ; K_{k_{1}-1}, k_{1}\right)$ and set $p_{3}=p_{2}^{\left(\mathbf{v}^{*}\right)}$. Then $p_{3}$ is $\left(K_{k}, k\right)$-fixed and $\left(K_{k}, k+1\right)$-fixed for every $k \geq k_{1}$, and

whenever $k \geq k_{1}, \mathbf{u} \in \mathbf{U}\left(p_{3} ; K_{k}, k\right)$.

$$
\mathcal{T}_{\mathbf{u}}=\left\{\mathbf{c}: \mathbf{u}^{\wedge} \mathbf{c} \in \mathbf{U}\left(p_{3} ; K_{k}, k+1\right)\right\}
$$

(d) We have 


$$
p_{3} \Vdash_{\mathbb{P}} \sigma(i)=H_{i}\left(\mathbf{v}_{i}^{*}\right) \forall i<k_{1}
$$

where $\mathbf{v}_{i}^{*}$ is that member of $T_{i+1}^{K_{i}}$ such that $\mathbf{v}_{i}^{*}(\eta) \leq \mathbf{v}^{*}(\eta)$ for every $\eta \in K_{i}$. Set $L_{k}^{\prime}=\left\{H_{k}\left(\mathbf{v}_{k}^{*}\right)\right\}$ for $k<k_{1}$, $L_{k}^{\prime}=L_{k} \backslash \tilde{J}_{k}$ for $k \geq k_{1}$; then $\prod_{k \in \mathbb{N}} \#\left(L_{k}^{\prime}\right) / n_{k}>0$, because $\prod_{k \in \mathbb{N}} \#\left(L_{k}\right) / n_{k}>0$ and $\sum_{k \in \mathbb{N}} \#\left(\tilde{J}_{k}\right) / n_{k}<\infty$. So there is a $\tilde{z} \in D \cap\left(\prod_{k \in \mathbb{N}} L_{k}^{\prime}\right)^{r} \subseteq D^{\prime}$. Writing $z_{k}=\langle\tilde{z}(j)(k)\rangle_{j<r}$ for $k \in \mathbb{N}$, we have $z_{k} \in\left(n_{k} \backslash \tilde{J}_{k}\right)^{r}$ for $k \geq k_{1}$, and

for $k<k_{1}$, because $(i, i) \in W_{k}$ for $i<n_{k}$.

$$
p_{3} \Vdash_{\mathbb{P}}\left(\sigma(k), z_{k}\right) \in \tilde{W}_{k}
$$

(e) For each $k \geq k_{1}, \mathbf{u} \in \mathbf{U}\left(p_{3} ; K_{k}, k\right)$ choose $\mathcal{T}_{\mathbf{u}}^{\prime} \preccurlyeq \mathcal{T}_{\mathbf{u}} \in \mathbb{T}_{k}$ such that either $H_{k}\left(\mathbf{u}^{\wedge} \mathbf{c}\right)=w_{\mathbf{u}} \in \tilde{I}_{k}$ for every $\mathbf{c} \in \mathcal{T}_{\mathbf{u}}^{\prime}$ or $\left(H_{k}\left(\mathbf{u}^{\wedge} \mathbf{c}\right), z_{k}\right) \in \tilde{W}_{k}$ for every $\mathbf{c} \in \mathcal{T}_{\mathbf{u}}^{\prime}$.

Define $\left\langle\mathcal{S}_{k}\right\rangle_{k \geq k_{1}},\left\langle\tilde{p}_{k}\right\rangle_{k \geq k_{1}}$ by

$$
\begin{aligned}
& \tilde{p}_{k_{1}}=p_{3}, \\
& \mathcal{S}_{k}=\left\{\mathbf{u}^{\wedge} \mathbf{c}: \mathbf{u} \in \mathbf{U}\left(\tilde{p}_{k} ; K_{k}, k\right), \mathbf{c} \in \mathcal{T}_{\mathbf{u}}^{\prime}\right\}, \\
& \tilde{p}_{k+1}=\bigvee\left\{\tilde{p}_{k}^{(\mathbf{v})}: \mathbf{v} \in \mathcal{S}_{k}\right\}
\end{aligned}
$$

for every $k \geq k_{1}$, as in Lemma $1 \mathrm{P}$. An easy induction on $k$ shows that

$$
\mathcal{T}_{\mathbf{u}}^{\prime}=\left\{\mathbf{c}: \mathbf{u}^{\wedge} \mathbf{c} \in \mathbf{U}\left(\tilde{p}_{k+1} ; K_{k}, k+1\right)\right\}
$$

whenever $\mathbf{u} \in \mathbf{U}\left(\tilde{p}_{k} ; K_{k}, k\right), k \geq k_{1}$, that $\tilde{p}_{k}$ is $\left(K_{l}, l\right)$-fixed and $\left(K_{l}, l+1\right)$-fixed whenever $k_{1} \leq k \leq l$, and that $\tilde{p}_{k}^{(\mathbf{v})}=p_{3}^{(\mathbf{v})}$ whenever $k_{1} \leq k \leq l$ and $\mathbf{v} \in \mathbf{U}\left(\tilde{p}_{k} ; K_{l}, l\right) \cup \mathbf{U}\left(\tilde{p}_{k} ; K_{l}, l+1\right)$. Also $\operatorname{supp}\left(\tilde{p}_{k}\right) \subseteq \bigcup_{l \in \mathbb{N}} K_{l}$ for every $k \geq k_{1}$.

(f) It is likewise easy to see that, for $k \geq k_{1}$,

$$
\begin{aligned}
& \tilde{p}_{k+1} \leq \tilde{p}_{k}, \\
& \tilde{p}_{k+1}\left\lceil\xi \Vdash_{\mathbb{P}_{\xi}} \tilde{p}_{k+1}(\xi) \cap T_{k}=\tilde{p}_{k}(\xi) \cap T_{k} \forall \xi<\kappa,\right.
\end{aligned}
$$$$
\tilde{p}_{k+1} \mid \xi \Vdash_{\mathbb{P}_{\xi}} \tilde{p}_{k+1}(\xi) \cap \operatorname{suc}(t)=\tilde{p}_{k}(\xi) \cap \operatorname{suc}(t) \forall t \in \tilde{p}_{k+1}(\xi) \cap T_{i}
$$

unless $i=k$ and $\xi \in K_{k}$,

$$
\tilde{p}_{k+1} \Vdash_{\mathbb{P}} \sigma(k) \in \tilde{I}_{k} \text { or }\left(\sigma(k), z_{k}\right) \in \tilde{W}_{k} .
$$

(g) On the other hand, if $k \geq k_{1}$ and $\xi \in K_{k}$,

$$
\begin{gathered}
\tilde{p}_{k+1}\left\lceil\xi \Vdash_{\mathbb{P}_{\xi}} \operatorname{dp}\left(\left\{C: t \times C \in \tilde{p}_{k+1}(\xi)\right\}\right) \geq \operatorname{dp}\left(\left\{C: t \times C \in \tilde{p}_{k}(\xi)\right\}\right) / 2 l_{k}\right. \\
\forall t \in \tilde{p}_{k+1}(\xi) \cap T_{k} .
\end{gathered}
$$

To see this, take any $q \leq \tilde{p}_{k+1}\lceil\xi$ and $t$ such that

$$
q \Vdash_{\mathbb{P}_{\xi}} t \in \tilde{p}_{k+1}(\xi) \cap T_{k}=\tilde{p}_{k}(\xi) \cap T_{k} .
$$

We may suppose that $\mathbf{v}_{0} \in \mathcal{S}_{k}$ is such that $q \leq \tilde{p}_{k}^{\left(\mathbf{v}_{0}\right)}\left\lceil\xi=\tilde{p}_{k+1}^{\left(\mathbf{v}_{0}\right)}\left\lceil\xi=q_{1}\right.\right.$. Now $\tilde{p}_{k+1}$ is $\left(K_{k}, k+1\right)$-fixed so there must be a $t^{\prime} \geq t$ such that

$$
q_{1} \Vdash_{\mathbb{P}_{\xi}} t^{\prime} \in T_{k+1} \cap \tilde{p}_{k+1}(\xi) .
$$

There is accordingly a $\mathbf{v}_{1} \in \mathcal{S}_{k}$ such that $\mathbf{v}_{0}\left\lceil\xi=\mathbf{v}_{1}\left\lceil\xi\right.\right.$ and $\mathbf{v}_{1}(\xi)=t^{\prime}$. Express $\mathbf{v}_{1}$ as $\mathbf{u}^{\wedge} \mathbf{c}_{1}$ where $\mathbf{u} \in$ $\mathbf{U}\left(\tilde{p}_{k} ; K_{k}, k\right)$ and $\mathbf{c}_{1} \in \mathcal{T}_{\mathbf{u}}^{\prime}$. Of course $\mathbf{u}(\xi)=t$.

Now

$$
\begin{aligned}
& q_{1} \Vdash_{\mathbb{P}_{\xi}}\left\{C: t \times C \in \tilde{p}_{k+1}(\xi)\right\} \supseteq\left\{\mathbf{c}(\xi): \mathbf{c} \in \mathcal{T}_{\mathbf{u}}^{\prime}, \mathbf{c}\left\lceil\xi=\mathbf{c}_{1}\lceil\xi\},\right.\right. \\
& q_{1} \Vdash_{\mathbb{P}_{\xi}}\left\{C: t \times C \in \tilde{p}_{k}(\xi)\right\}=\left\{\mathbf{c}(\xi): \mathbf{c} \in \mathcal{T}_{\mathbf{u}}, \mathbf{c}\left\lceil\xi=\mathbf{c}_{1}\lceil\xi\}\right.\right.
\end{aligned}
$$

because $\tilde{p}_{k}$ and $\tilde{p}_{k+1}$ are both $\left(K_{k}, k+1\right)$-fixed, while $\mathbf{v}_{0}\left\lceil\xi=\left(\mathbf{u}\lceil\xi)^{\wedge}\left(\mathbf{c}_{1} \mid \xi\right)\right.\right.$. But because $\mathcal{T}_{\mathbf{u}}^{\prime} \preccurlyeq k \mathcal{T}_{\mathbf{u}}$,

So we get

$$
\operatorname{dp}\left(\left\{\mathbf{c}(\xi): \mathbf{c} \in \mathcal{T}_{\mathbf{u}}^{\prime}, \mathbf{c}\left\lceil\xi=\mathbf{c}_{1}\lceil\xi\}\right) \geq \operatorname{dp}\left(\left\{\mathbf{c}(\xi): \mathbf{c} \in \mathcal{T}_{\mathbf{u}}, \mathbf{c}\left\lceil\xi=\mathbf{c}_{1} \mid \xi\right\}\right) / 2 l_{k} .\right.\right.\right.
$$

As $q$ and $t$ are arbitrary, we have the result.

(h) Because $\gamma_{k} \ln \left(2 l_{k}\right) \leq 2^{-k}($ by $1 \mathrm{E}(\mathrm{a})(\mathrm{v}))$,

$$
\tilde{p}_{k+1} \leq_{K_{k}, k} \tilde{p}_{k}
$$

for every $k \geq k_{1}$. Also, $\operatorname{supp}\left(\tilde{p}_{k}\right) \subseteq \bigcup_{l \in \mathbb{N}} K_{l}$ for every $k \geq k_{1}$. By Lemma $1 \mathrm{~N}$, there is a $p_{4} \in \mathbb{P}$ such that $p_{4} \leq \tilde{p}_{k}$ for every $k \geq k_{1}$. Moreover, we may take it that

(as in Lemma 1H), so that

$$
p_{4}(0)=\bigcap_{k \geq k_{1}} \tilde{p}_{k}(0)
$$


whenever $i \geq k_{1}$. Note that for $k \geq k_{1}$,

$$
\delta_{i}\left(p_{4}(0)\right) \geq \delta_{i}\left(p_{3}(0)\right)-\gamma_{i} \ln \left(2 l_{i}\right) \geq 1
$$

while for $k<k_{1}$,

$$
p_{4} \Vdash_{\mathbb{P}} \sigma(k) \in \tilde{I}_{k} \text { or }\left(\sigma(k), z_{k}\right) \in \tilde{W}_{k},
$$

$$
p_{4} \Vdash_{\mathbb{P}}\left(\sigma(k), z_{k}\right) \in \tilde{W}_{k} .
$$

(i) Now define $p_{5} \in \mathbb{P}$ by setting

$$
\begin{aligned}
& \tilde{I}_{i}^{\prime}=\tilde{I}_{i} \cup\{\tilde{z}(j)(i): j<r\} \forall i \in \mathbb{N}, \\
& p_{5}(0)=\left\{t: t \in p_{4}(0), C_{i}(t) \cap \tilde{I}_{i}^{\prime}=\emptyset \text { whenever } k_{1} \leq i<\operatorname{rank}(t)\right\},
\end{aligned}
$$$$
p_{5}(\xi)=p_{4}(\xi) \text { if } 0<\xi<\kappa \text {. }
$$

Of course we must check that $p_{5}(0)$, as so defined, belongs to $\mathbb{Q}_{0} \cong \mathbb{Q}$; but because $\delta_{k}\left(p_{4}(0)\right) \geq 1$ for $k \geq k_{1}$, we have

$$
\operatorname{dp}\left(\left\{C: t \times C \in p_{4}(0)\right\}\right) \geq \exp \left(1 / \gamma_{k}\right) \geq 2\left(\prod_{i<k} 2^{n_{i}}\right)^{k+1}+2 k \geq 2 \#\left(\tilde{I}_{k}^{\prime}\right)
$$

for every $k \geq k_{1}, t \in p_{4}(0) \cap T_{k}$, using $1 \mathrm{E}(\mathrm{a})\left(\right.$ vii). Of course $p_{5}(0) \cap T_{k_{1}-1}=p_{4}(0) \cap T_{k_{1}-1}=\left\{\mathbf{v}^{*}(0)\right\}$, so every element of $p_{5}(0)$ has a successor in $p_{5}(0)$, and also

$$
\delta_{i}\left(p_{5}(0)\right) \geq \delta_{i}\left(p_{4}(0)\right)-\gamma_{i} \ln 2
$$

for $i \geq k_{1}$. (Here at last is the key step which depends on using dp in our measure of 'rapidly branching' given in $1 \mathrm{E}(\mathrm{d})$.) Thus $p_{5}(0) \in \mathbb{Q}$ and $p_{5} \in \mathbb{P}$. But also

so that

$$
p_{5} \Vdash_{\mathbb{P}} \Phi_{k} \cap \tilde{I}_{k}^{\prime}=\emptyset \forall k \geq k_{1},
$$

for $k \geq k_{1}$; finally

$$
p_{5} \Vdash_{\mathbb{P}} \sigma(k) \notin \tilde{I}_{k}, \sigma(k) \neq \tilde{z}(j)(k) \forall j<r,\left(\sigma(k), z_{k}\right) \in \tilde{W}_{k}
$$

as $p_{5} \leq p$ and $p, \sigma$ are arbitrary,

$$
p_{5} \Vdash_{\mathbb{P}}(\sigma, \tilde{z}) \in\left\ulcorner R_{r}\right\urcorner, \sigma \in\left\ulcorner R_{r}\right\urcorner-1\left[D^{\prime}\right] ;
$$

as claimed.

$$
\mathbb{1}_{\mathbb{P}} \Vdash_{\mathbb{P}} \Psi \cap \prod_{k \in \mathbb{N}} L_{k} \subseteq\left\ulcorner R_{r}\right\urcorner^{-1}\left[D^{\prime}\right],
$$

1S Theorem For each $r \in \mathbb{N}$,

$$
\begin{gathered}
\mathbb{1}_{\mathbb{P}} \Vdash_{\mathbb{P}} \text { if } D_{i} \subseteq\ulcorner X\urcorner \text { and } D_{i} \cap \prod_{k \in \mathbb{N}} L_{k} \neq \emptyset \forall\left\langle L_{k}\right\rangle_{k \in \mathbb{N}} \in\ulcorner\mathcal{L}\urcorner, i<r, \\
\text { then } \forall\left\langle L_{k}\right\rangle_{k \in \mathbb{N}} \in\ulcorner\mathcal{L}\urcorner \exists\left\langle x_{i}\right\rangle_{i<r} \in \prod_{i<r}\left(D_{i} \cap \prod_{k \in \mathbb{N}} L_{k}\right) \\
\text { such that }\left(x_{j}, x_{i}\right) \in\ulcorner R\urcorner \forall i<j<r .
\end{gathered}
$$

proof Induce on $r$. If $r=0$ the result is trivial. For the inductive step to $r+1$, take $\mathbb{P}$-names $\Delta_{i}$ for subsets of $\ulcorner X\urcorner$ such that

$$
\mathbb{1}_{\mathbb{P}} \Vdash_{\mathbb{P}} \Delta_{i} \cap \prod_{k \in \mathbb{N}} L_{k} \neq \emptyset \forall\left\langle L_{k}\right\rangle_{k \in \mathbb{N}} \in\ulcorner\mathcal{L}\urcorner, i \leq r .
$$

Take a $\mathbb{P}$-name $\mathfrak{L}$ for a member of $\ulcorner\mathcal{L}\urcorner$. Because members of $\mathcal{L}$ can be coded by simple sequences, we may suppose that $\mathfrak{L}$ is a $\mathbb{P}_{\alpha}$-name for some $\alpha<\kappa$ (1Je). The inductive hypothesis tells us that

$$
\mathbb{1}_{\mathbb{P}} \Vdash_{\mathbb{P}} \forall\left\langle L_{k}\right\rangle_{k \in \mathbb{N}} \in\ulcorner\mathcal{L}\urcorner \exists\left\langle x_{i}\right\rangle_{i<r} \in \prod_{i<r}\left(\Delta_{i} \cap \prod_{k \in \mathbb{N}} L_{k}\right)
$$

$$
\text { such that }\left(x_{j}, x_{i}\right) \in\ulcorner R\urcorner \text { if } i<j<r \text {. }
$$

Next, using 1 Jc-e, we can find a $\beta \in \kappa \backslash \alpha$ and a $\mathbb{P}_{\beta}$-name $\Delta$ for a subset of $\left\ulcorner X^{r}\right\urcorner$ such that

$$
\mathbb{1}_{\mathbb{P}} \Vdash_{\mathbb{P}} \Delta \subseteq \prod_{i<r} \Delta_{i},
$$

$$
\begin{array}{r}
\mathbb{1}_{\mathbb{P}_{\beta}} \Vdash_{\mathbb{P}_{\beta}}\left(x_{j}, x_{i}\right) \in\ulcorner R\urcorner(\beta) \text { whenever }\left\langle x_{l}\right\rangle_{l<r} \in \Delta, i<j<r, \\
\Delta \cap\left(\prod_{k \in \mathbb{N}} L_{k}\right)^{r} \neq \emptyset \forall\left\langle L_{k}\right\rangle_{k \in \mathbb{N}} \in\ulcorner\mathcal{L}\urcorner(\beta),
\end{array}
$$

where we write $\ulcorner\ldots\urcorner(\urcorner^{(\beta)}$ to indicate that we are interpreting some formula in $V^{\mathbb{P}_{\beta}}$. 
Now we remark that by $1 \mathrm{Jb} \mathbb{P}$ can be regarded, for forcing purposes, as an iteration $\mathbb{P}_{\beta} * \mathbb{P}^{\prime}$, where $\mathbb{P}^{\prime}$ is a $\mathbb{P}_{\beta}$-name for a p.o.set with the same definition, interpreted in $V^{\mathbb{P}_{\beta}}$, as $\mathbb{P}$ has in the ground model. So we may use Lemma $1 \mathrm{R}$ in $V^{\mathbb{P}_{\beta}}$ to say that

$$
\mathbb{1}_{\mathbb{P}_{\beta}} \Vdash_{\mathbb{P}_{\beta}}\left(\mathbb{1}_{\mathbb{P}^{\prime}} \Vdash_{\mathbb{P}^{\prime}} \Psi^{(\beta)} \cap \prod \mathfrak{L} \subseteq\left\ulcorner R_{r}\right\urcorner^{-1}\left[\Delta \cap\left(\prod \mathfrak{L}\right)^{r}\right]\right),
$$

using the notation $\Psi^{(\beta)}$ to indicate which version of the $\mathbb{P}$-name $\Psi$ we are trying to use. Moving to $V^{\mathbb{P}}$ for a moment, we have $\ulcorner\mu\urcorner \Psi^{(\beta)}=1$ and $\ulcorner\mu\urcorner\left(\prod \mathfrak{L}\right)>0$, so

$$
\mathbb{1}_{\mathbb{P}} \Vdash_{\mathbb{P}} \exists l \in \mathbb{N},\ulcorner\mu\urcorner\left(\Psi_{l}^{(\beta)} \cap \prod \mathfrak{L}\right)>0 .
$$

Also, of course, every $\Psi_{l}^{(\beta)} \cap \prod \mathfrak{L}$ can be regarded (in $V^{\mathbb{P}}$ ) as the product of a sequence belonging to $\ulcorner\mathcal{L}\urcorner$. By the original hypothesis on $\Delta_{r}$,

$$
\mathbb{1}_{\mathbb{P}} \Vdash_{\mathbb{P}} \Delta_{r} \cap \Psi^{(\beta)} \cap \prod \mathfrak{L} \neq \emptyset .
$$

We can therefore find a $\mathbb{P}$-name $\sigma_{r}$ for a member of $\Delta_{r} \cap \Psi^{(\beta)} \cap \prod \mathfrak{L}$, and now further $\mathbb{P}_{\beta}$-names $\sigma_{i}$, for $i<r$, such that

But of course we now have

$$
\mathbb{1}_{\mathbb{P}} \Vdash_{\mathbb{P}}\left\langle\sigma_{i}\right\rangle_{i<r} \in \Delta \cap\left(\prod \mathfrak{L}\right)^{r},\left(\sigma_{r},\left\langle\sigma_{i}\right\rangle_{i<r}\right) \in\left\ulcorner R_{r}\right\urcorner .
$$

$$
\mathbb{1}_{\mathbb{P}} \Vdash_{\mathbb{P}}\left\langle\sigma_{i}\right\rangle_{i \leq r} \in \prod_{i \leq r}\left(\Delta_{i} \cap \prod \mathfrak{L}\right),\left(\sigma_{j}, \sigma_{i}\right) \in\ulcorner R\urcorner \forall i<j \leq r .
$$

As $\left\langle\Delta_{i}\right\rangle_{i \leq r}, \mathfrak{L}$ are arbitrary, this shows that the induction proceeds.

Version of 6.8 .91

2. Pointwise compact sets of measurable functions We turn now to the questions in analysis which the construction in $\S 1$ is designed to solve. We begin with some definitions and results taken from [Ta84].

2A Definitions (a) Let $(X, \Sigma, \mu)$ be a probability space. Write $\mathcal{L}^{0}=\mathcal{L}^{0}(\Sigma) \subseteq \mathbb{R}^{X}$ for the set of $\Sigma$ measurable real-valued functions on $X$. Let $\mathfrak{T}_{p}$ be the topology of pointwise convergence, the usual product topology, on $\mathbb{R}^{X}$. Let $\mathfrak{T}_{m}$ be the (non-Hausdorff, non-locally-convex) topology of convergence in measure on $\mathcal{L}^{0}$, defined by the pseudometric

for $f, g \in \mathcal{L}^{0}$.

$$
\rho(f, g)=\int \min (|f(x)-g(x)|, 1) \mu(d x)
$$

(b) A set $A \subseteq \mathbb{R}^{X}$ is stable if whenever $\alpha<\beta$ in $\mathbb{R}, E \in \Sigma$ and $\mu E>0$ there are $k, l \geq 1$ such that $\mu_{k+l}^{*}\left\{(\mathbf{x}, \mathbf{y}): \mathbf{x} \in E^{k}, \mathbf{y} \in E^{l}, \exists f \in A, f(\mathbf{x}(i)) \leq \alpha \& f(\mathbf{y}(j)) \geq \beta \forall i<k, j<l\right\}$

$$
<(\mu E)^{k+l},
$$

writing $\mu_{k+l}^{*}$ for the usual product outer measure on $X^{k} \times X^{l}$. (See [Ta84], 9-1-1.)

2B Stable sets Suppose that $(X, \Sigma, \mu)$ is a probability space and that $A \subseteq \mathbb{R}^{X}$ is a stable set.

(a) If $(X, \Sigma, \mu)$ is complete, then $A \subseteq \mathcal{L}^{0}(\Sigma)$. ([Ta84], §9.1.)

(b) The $\mathfrak{T}_{p}$-closure of $A$ in $\mathbb{R}^{X}$ is stable.

(c) If $A$ is bounded above and below by members of $\mathcal{L}^{0}$, its convex hull is stable ([Ta84], 11-2-1).

(d) If $A \subseteq \mathcal{L}^{0}$ (as in (a)), then $\mathfrak{T}_{m}\left\lceil A\right.$, the subspace topology on $A$ induced by $\mathfrak{T}_{m}$, is coarser than $\mathfrak{T}_{p}\lceil A$. ([Ta84], 9-5-2.)

For more about stable sets, see [Ta84]and [Ta87].

2C Pettis integration Let $(X, \Sigma, \mu)$ be a probability space and $B$ a (real) Banach space.

(a) A function $\phi: X \rightarrow B$ is scalarly measurable if $g \phi: X \rightarrow \mathbb{R}$ is $\Sigma$-measurable for every $g \in B^{*}$, the continuous dual of $B$.

(b) In this case, $\phi$ is Pettis integrable if there is a function $\theta: \Sigma \rightarrow B$ such that

$$
\int_{E} g \phi d \mu \text { exists }=g(\theta E) \forall E \in \Sigma, g \in B^{*} .
$$

(c) If $\phi: X \rightarrow B$ is bounded and scalarly measurable, then

$$
A=\left\{g \phi: g \in B^{*},\|g\| \leq 1\right\} \subseteq \mathcal{L}^{0}
$$

is $\mathfrak{T}_{p}$-compact. In this case $\phi$ is Pettis integrable iff

$$
f \mapsto \int_{E} f: A \rightarrow \mathbb{R}
$$


is $\mathfrak{T}_{p}\lceil A$-continuous for every $E \in \Sigma$ ([Ta84], 4-2-3). In particular (by $2 \mathrm{~B}(\mathrm{~d})) \phi$ is Pettis integrable if $A$ is stable.

2D The rivals Write $\mu_{L}$ for Lebesgue measure on $[0,1]$, and $\Sigma_{L}$ for its domain. Consider the following two propositions:

$\left.{ }^{*}\right)[0,1]$ is not the union of fewer than $\mathfrak{c}$ closed negligible sets;

$(\dagger)$ there are sequences $\left\langle n_{k}\right\rangle_{k \in \mathbb{N}},\left\langle W_{k}\right\rangle_{k \in \mathbb{N}}$ such that

$n_{k} \geq 2^{k}, W_{k} \subseteq n_{k} \times n_{k}, \#\left(W_{k}\right) \leq 2^{-k} n_{k}^{2} \forall k \in \mathbb{N} ;$

taking $X=\prod_{k \in \mathbb{N}} n_{k}, \mu$ the usual Radon probability on $X$,

$$
R=\left\{(x, y): x, y \in X,(x(k), y(k)) \in W_{k} \forall k \in \mathbb{N},\{k: x(k)=y(k)\} \text { is finite }\right\},
$$

then whenever $D \subseteq X, \mu^{*} D=1$ and $r \in \mathbb{N}$ there are $x_{0}, \ldots, x_{r} \in D$ such that $\left(x_{j}, x_{i}\right) \in R$ whenever

$i<j \leq r$.

Evidently $\left(^{*}\right)$ is a consequence of $\mathrm{CH}$, while in the language of $\S 1, \mathbb{1}_{\mathbb{P}} \Vdash_{\mathbb{P}}(\dagger)$, this being a slightly weaker version of Theorem $1 \mathrm{~S}$.

Thus both $\left(^{*}\right)$ and $(\dagger)$ are relatively consistent with ZFC. Consequences of $\left(^{*}\right)$ are explored in [Ta84], where it is called Axiom L; we list a few of them in $2 \mathrm{E}$ below. Our purpose in this paper is to show that $(\dagger)$ leads to a somewhat different world.

2E Theorem Assume $\left.{ }^{*}\right)$. Write $\mathcal{L}^{0}$ for $\mathcal{L}^{0}\left(\Sigma_{L}\right)$.

(a) If $A \subseteq \mathcal{L}^{0}$ is separable and compact for $\mathfrak{T}_{p}$, it is stable.

(b) If $A \subseteq \mathcal{L}^{0}$ is separable and compact for $\mathfrak{T}_{p}$, its closed convex hull in $\mathbb{R}^{[0,1]}$ lies within $\mathcal{L}^{0}$.

(c) If $A \subseteq \mathcal{L}^{0}$ is separable and compact for $\mathfrak{T}_{p}$, then $\mathfrak{T}_{m}\left\lceil A\right.$ is coarser than $\mathfrak{T}_{p}\lceil A$.

(d) If $(Y, \mathfrak{S}, \mathrm{T}, \nu)$ is a separable compact Radon measure space and $f:[0,1] \times Y \rightarrow \mathbb{R}$ is measurable in the first variable and continuous in the second, then it is measurable for the (completed) product measure $\mu_{L} \times \nu$.

(e) If $\left\langle E_{n}\right\rangle_{n \in \mathbb{N}}$ is a stochastically independent sequence of measurable subsets of [0,1], with $\lim _{n \rightarrow \infty} \mu E_{n}=$ 0 but $\sum_{n \in \mathbb{N}}\left(\mu E_{n}\right)^{k}=\infty$ for every $k \in \mathbb{N}$, then there is an ultrafilter $\mathcal{F}$ on $\mathbb{N}$ such that

is non-measurable.

$$
\lim _{n \rightarrow \mathcal{F}} E_{n}=\left\{x:\left\{n: x \in E_{n}\right\} \in \mathcal{F}\right\}
$$

proof (a) See [Ta84], 9-3-1(b). (b) Use (a) and 2B(c). (c) Use (a) and 2B(d). (d) Use (a) and [Ta84], 10-2-1. (e) Observe that, writing $\chi E_{n}$ for the characteristic function of $E_{n}$, the set $\left\{\chi E_{n}: n \in \mathbb{N}\right\}$ is not stable, and use (a).

2F Theorem Assume $(\dagger)$.

(a) There is a bounded Pettis integrable function $\phi:[0,1] \rightarrow \ell^{\infty}$ such that $\left\{g \phi: g \in\left(\ell^{\infty}\right)^{*},\|g\| \leq 1\right\}$ is not stable in $\mathcal{L}^{0}\left(\Sigma_{L}\right)$.

(b) There is a separable convex $\mathfrak{T}_{p^{-}}$-compact subset of $\mathcal{L}^{0}\left(\Sigma_{L}\right)$ which is not stable.

proof (We write $\ell^{\infty}$ for the Banach space of bounded real sequences.) Take $\left\langle n_{k}\right\rangle_{k \in \mathbb{N}},\left\langle W_{k}\right\rangle_{k \in \mathbb{N}}, X, \mu, R$ from the statement of $(\dagger)$. Because $\left([0,1], \mu_{L}\right)$ is isomorphic, as measure space, to $(X, \mu)$, we may work with $X$ rather than with $[0,1]$. Write $\Sigma$ for the domain of $\mu, \mathcal{L}^{0}=\mathcal{L}^{0}(\Sigma)$.

(a) For $k \in \mathbb{N}$ write

For $k \in \mathbb{N}, I \subseteq n_{k}$ set

$$
\mathcal{I}_{k}=\left\{I: I \subseteq n_{k}, \#(I) \leq k,(i, j) \notin W_{k} \text { for all distinct } i, j \in I\right\},
$$

Let $A$ be

$$
H_{k I}=\{x: x \in X, x(k) \in I\} .
$$

$$
\left\{\chi H_{k I}: k \in \mathbb{N}, I \in \mathcal{I}_{k}\right\},
$$

writing $\chi H: X \rightarrow\{0,1\}$ for the characteristic function of $H \subseteq X$; let $Z$ be the $\mathfrak{T}_{p}$-closure of $A$ in $\mathbb{R}^{X}$. Because $A$ is uniformly bounded, $Z$ is $\mathfrak{T}_{p}$-compact. For $E \in \Sigma$ define $f_{E}: Z \rightarrow \mathbb{R}$ by setting $f_{E}(h)=\int_{E} h(x) \mu(d x)$ for $h \in A, f_{E}(u)=0$ for $u \in Z \backslash A$. Enumerate $A$ as $\left\langle h_{m}\right\rangle_{m \in \mathbb{N}}$, and define $\phi: X \rightarrow \ell^{\infty}, \theta: \Sigma \rightarrow \ell^{\infty}$ by setting

$$
\begin{gathered}
\phi(x)(m)=h_{m}(x) \forall m \in \mathbb{N}, x \in X, \\
\theta(E)(m)=\int_{E} h_{m}(x) \mu(d x) \forall m \in \mathbb{N}, E \in \Sigma .
\end{gathered}
$$

We aim to show 
(i) that $A$ is not stable;

(ii) that if $\nu$ is a Radon probability on $A^{\prime}=Z \backslash A$ then $\int u(x) \nu(d u)=0$ for $\mu$-almost every $x$;

(iii) $f_{E}: Z \rightarrow \mathbb{R}$ is continuous for every $E \in \Sigma$;

(iv) $\theta$ is the indefinite Pettis integral of $\phi$, so that $\phi$ is Pettis integrable;

(v) $K=\left\{g \phi: g \in C(Z)^{*},\|g\| \leq 1\right\}$ includes $A$ so is not stable.

ad (i) Suppose that $k, l \geq 1$. Take any $m \geq l$. Set

$$
\begin{aligned}
G & =\left\{\mathbf{y}: \mathbf{y} \in X^{l}, \exists I \in \mathcal{I}_{m}, \mathbf{y}(j) \in H_{m I} \forall j<l\right\} \\
& \supseteq\left\{\mathbf{y}: \mathbf{y} \in X^{l},(\mathbf{y}(i)(m), \mathbf{y}(j)(m)) \notin W_{m} \text { for distinct } i, j<l\right\} .
\end{aligned}
$$

Because $\#\left(W_{m}\right) \leq 2^{-m} n_{m}^{2}, \mu_{l} G \geq\left(1-2^{-m}\right)^{l^{2}}$. If $\mathbf{y} \in G$, set $I=\{\mathbf{y}(j)(m): j<l\} \in \mathcal{I}_{m}$; then

So we conclude that

$$
\mu_{k}\left\{\mathbf{x}: \mathbf{x} \in X^{k}, \mathbf{x}(i)(m) \notin I \forall i<k\right\} \geq\left(1-n_{m}^{-1} l\right)^{k} .
$$

$$
\begin{gathered}
\mu_{k+l}\left\{(\mathbf{x}, \mathbf{y}): \mathbf{x} \in X^{k}, \mathbf{y} \in X^{l}, \exists f \in A, f(\mathbf{x}(i))=0 \forall i<k, f(\mathbf{y}(j))=1 \forall j<l\right\} \\
\geq\left(1-2^{-m}\right)^{l^{2}}\left(1-n_{m}^{-1} l\right)^{k}
\end{gathered}
$$

(by Fubini's theorem). Because $k, l$ and $m$ are arbitrary, $A$ cannot be stable.

ad (ii) Because each $\mathcal{I}_{m}$ is finite, any member of $A^{\prime}$ must be of the form $\chi E$ where $E \subseteq X$ and $x \in E, x^{\prime} \in X,\left\{k: x(k) \neq x^{\prime}(k)\right\}$ is finite $\Rightarrow x^{\prime} \in E$.

Note also that if $x, y \in E$ then $(x, y) \notin R$; because either $x(k)=y(k)$ for infinitely many $k$, or there are $k$, $I$ such that $x(k) \neq y(k), I \in \mathcal{I}_{k}$ and $x, y$ both belong to $H_{k I}$, in which case $(x(k), y(k)) \notin W_{k}$.

Now let $\nu$ be a Radon probability on $A^{\prime}$, and set $w(x)=\int u(x) \nu(d u)$ for each $x \in X$, so that $w$ belongs to the closed convex hull of $A^{\prime}$ in $\mathbb{R}^{X}$. If $x, x^{\prime}$ are two members of $X$ differing on only finitely many coordinates, then $u(x)=u\left(x^{\prime}\right)$ for every $u \in A^{\prime}$; consequently $w(x)=w\left(x^{\prime}\right)$. Also $0 \leq w(x) \leq 1$ for every $x \in X$.

Take $\delta>0$ and set $D=\{x: w(x) \geq \delta\}$. By the zero-one law, $\mu^{*} D$ must be either 0 or 1 . Suppose, if possible, that $\mu^{*} D=1$. Let $r \in \mathbb{N}$ be such that $r \delta \geq 1$. By $(\dagger)$, there are $x_{0}, \ldots, x_{r} \in D$ such that $\left(x_{j}, x_{i}\right) \in R$ for $i<j \leq r$. But in this case $\sum_{i \leq r} u\left(x_{i}\right) \leq 1$ for every $u \in A^{\prime}$, while $\sum_{i \leq r} w\left(x_{i}\right) \geq(r+1) \delta>1$, and $w$ cannot belong to the closed convex hull of $A^{\prime}$.

Accordingly $\mu^{*} D$ must be 0 . As $\delta$ is arbitrary, $w=0$ a.e.

ad (iii) Because $f_{E}\left(\chi H_{k I}\right) \leq k n_{k}^{-1}$ for every $I \in \mathcal{I}_{k}, \lim _{m \rightarrow \infty} f_{E}\left(h_{m}\right)=0$ and $f_{E}$ is continuous.

ad (iv) We need to show that

$$
\int_{E} g(\phi(x)) \mu(d x) \text { exists }=g(\theta(E)) \forall g \in\left(\ell^{\infty}\right)^{*}, E \in \Sigma .
$$

It is enough to consider positive linear functionals $g$ of norm 1 . For any such $g$ we have a Radon probability $\nu$ on $Z$ such that

$$
g\left(\left\langle f\left(h_{m}\right)\right\rangle_{m \in \mathbb{N}}\right)=\int_{Z} f(u) \nu(d u) \text { for every } f \in C(Z),
$$

using the Riesz representation of positive linear functionals on $C(Z)$. Set $\epsilon_{m}=\nu\left\{h_{m}\right\}, \epsilon=1-\sum_{m \in \mathbb{N}} \epsilon_{m}=$ $\nu A^{\prime}$. Then we can find a Radon probability $\nu^{\prime}$ on $A^{\prime}$ such that

$$
g\left(\left\langle f\left(h_{m}\right)\right\rangle_{m \in \mathbb{N}}\right)=\sum_{m \in \mathbb{N}} \epsilon_{m} f\left(h_{m}\right)+\epsilon \int_{A^{\prime}} f(u) \nu^{\prime}(d u)
$$

for every $f \in C(Z)$. Now an easy calculation (using (ii)) shows that

for every $E \in \Sigma$.

$$
g(\theta(E))=g\left(\left\langle f_{E}\left(h_{m}\right)\right\rangle_{m \in \mathbb{N}}\right)=\sum_{m \in \mathbb{N}} \epsilon_{m} \int_{E} h_{m}(x) \mu(d x)=\int_{E} g(\phi(x)) \mu(d x)
$$

ad (v) If $m \in \mathbb{N}$ then $h_{m}=e_{m} \phi \in K$ where $e_{m} \in\left(\ell^{\infty}\right)^{*}$ is defined by setting $e_{m}(z)=z(m)$ for every $z \in \ell^{\infty}$. This completes the proof.

(b) The unit ball of $\left(\ell^{\infty}\right)^{*}$ is $\mathrm{w}^{*}$-separable and its continuous image $K \subseteq \mathcal{L}^{0}$ is separable; so $K$ witnesses the truth of (b).

2G Further properties of the model Returning to $1 \mathrm{R} / 1 \mathrm{~S}$, we see that the model of $\S 1$ has some further striking characteristics closely allied to, but not obviously derivable from, $(\dagger)$. Consider for instance

$(\ddagger)$ there is a closed negligible set $Q \subseteq[0,1]^{2}$ such that whenever $D \subseteq[0,1]$ and $\mu_{L}^{*} D=1$ then $\mu_{L} Q^{-1}[D]=1$;

$(\ddagger)^{\prime}$ there is a negligible set $Q^{\prime} \subseteq[0,1]^{2}$ such that whenever $C, D \subseteq[0,1]$ and $(C \times D) \cap Q^{\prime}=\emptyset$ then one of $C, D$ is negligible. 
Then $\mathbb{1}_{\mathbb{P}} \Vdash_{\mathbb{P}}(\ddagger)$. For start by taking $Q_{1}$ to be

$$
\left\{(x, y): x, y \in X,(x(k), y(k)) \in W_{k} \forall k \in \mathbb{N}\right\},
$$

the closure of $R$ in $X \times X$. Then the argument for $1 \mathrm{~S}$ shows that

$$
\begin{gathered}
\mathbb{1}_{\mathbb{P}} \Vdash_{\mathbb{P}} \text { if } D \subseteq\ulcorner X\urcorner \text { and } D \cap \prod_{k \in \mathbb{N}} L_{k} \neq \emptyset \forall\left\langle L_{k}\right\rangle_{k \in \mathbb{N}} \in\ulcorner\mathfrak{L}\urcorner \\
\text { then } \exists \beta<\kappa \text { such that } \Psi^{(\beta)} \subseteq\left\ulcorner Q_{1}\right\urcorner^{-1}[D] .
\end{gathered}
$$

Consequently

$$
\mathbb{1}_{\mathbb{P}} \Vdash_{\mathbb{P}} \text { if } D \subseteq\ulcorner X\urcorner \text { and }\ulcorner\mu\urcorner^{*} D=1 \text { then }\ulcorner\mu\urcorner\left(\left\ulcorner Q_{1}\right\urcorner^{-1}[D]\right)=1 .
$$

Accordingly we have in $V^{\mathbb{P}}$ the version of $(\ddagger)$ in which $\left([0,1], \mu_{L}\right)$ is replaced by $(X, \mu)$. However there is now a continuous inverse-measure-preserving function $f: X \rightarrow[0,1]$, and taking

$$
Q=\left\{(f(x), f(y)):(x, y) \in Q_{1}\right\}
$$

we obtain ( $\ddagger$ ) itself. Evidently $(\ddagger)$ implies $(\ddagger)^{\prime}$, taking $Q^{\prime}$ to be

$$
\left\{\left(x+q, y+q^{\prime}\right):(x, y) \in Q, q, q^{\prime} \text { are rational }\right\} \cap[0,1]^{2} .
$$

Of course $\left(^{*}\right)$ and $(\ddagger)$ are mutually incompatible (the argument for $2 \mathrm{E}(\mathrm{a})$ from $\left(^{*}\right)$, greatly simplified, demolishes $(\ddagger)$ also). The weaker form $(\ddagger)^{\prime}$ is incompatible with CH or MA, but not with $\left(^{*}\right)$, both $(\ddagger)^{\prime}$ and $\left(^{*}\right)$ being true in Cohen's original model of not-CH (see [Frp89]).

2H Problems The remarkable results quoted in $2 \mathrm{E}$ depend on the identification of separable relatively pointwise compact sets with stable sets ('Axiom F' of [Ta84]). In models satisfying ( $\dagger$ ), this identification breaks down. But our analysis does not seem to touch any of $2 \mathrm{E}(\mathrm{b})-(\mathrm{e})$. We therefore spell out the obvious problems still outstanding. Write $\mathcal{L}^{0}$ for $\mathcal{L}^{0}\left(\Sigma_{L}\right)$.

(a) Is it relatively consistent with ZFC to suppose that there is a separable $\mathfrak{T}_{p^{-}}$-ompact set $A \subseteq \mathcal{L}^{0}$ such that the closed convex hull of $A$ in $\mathbb{R}^{[0,1]}$ does not lie within $\mathcal{L}^{0}$ ?

(b) Is it relatively consistent with ZFC to suppose that there is a separable $\mathfrak{T}_{p^{-}}$compact set $A \subseteq \mathcal{L}^{0}$ such that $\mathfrak{T}_{m}\left\lceil A\right.$ is not coarser than $\mathfrak{T}_{p}\lceil A$ ? Does it make a difference if $A$ is assumed to be convex? (This question seems first to have been raised by J.Bourgain and F.Delbaen.)

(c) Is it relatively consistent with ZFC to suppose that there are a separable compact Radon measure space $(Y, \mathfrak{S}, \mathrm{T}, \nu)$ and a function $f:[0,1] \times Y \rightarrow \mathbb{R}$ which is measurable in the first variable, continuous in the second variable, but not jointly measurable for $\mu_{L} \times \nu$ ?

(d) Is it relatively consistent with ZFC to suppose that there is a stochastically independent sequence $\left\langle E_{n}\right\rangle_{n \in \mathbb{N}}$ in $\Sigma_{L}$ such that $\sum_{n \in \mathbb{N}}\left(\mu_{L} E_{n}\right)^{k}=\infty$ for every $k \in \mathbb{N}$, but $\mu_{L}\left(\lim _{n \rightarrow \mathcal{F}} E_{n}\right)=0$ for every nonprincipal ultrafilter $\mathcal{F}$ on $\mathbb{N}$ ? (This question is essentially due to W.Moran; see also [Ta84], 9-1-4 for another version.)

Here we note only that a positive answer to (a) would imply the same answer to (c), and that the word 'separable' in (a)-(c) is necessary, as is shown by examples 3-2-3 and 10-1-1 in [Ta84].

\section{References}

[BJSp89] T.Bartoszyński, H.Judah \& S.Shelah, 'The Cichoń diagram', preprint, 1989 (MSRI 00626-90).

[Ba84] J.E.Baumgartner, 'Applications of the proper forcing axiom', pp. 913-959 in [KV84].

[Frp89] H.Friedman, 'Rectangle inclusion problems', Note of 9 October 1989.

[Ku80] K.Kunen, Set Theory. North-Holland, 1980.

[KV84] K.Kunen \& J.E.Vaughan (eds.), Handbook of Set-Theoretic Topology. North-Holland, 1984.

[Sh82] S.Shelah, Proper Forcing. Springer, 1982 (Lecture Notes in Mathematics 940).

[Sh326] S.Shelah, 'Vive la différence!', submitted for the proceedings of the set theory conference at MSRI, October 1989; notes of July 1987, preprint of October 1989; abbreviation 'ShCBF'.

[Sp87] J.Spencer, Ten Lectures on the Probabilistic Method, S.I.A.M., 1987.

[Ta84] M.Talagrand, Pettis integral and measure theory. Mem. Amer. Math. Soc. 307 (1984).

[Ta87] M.Talagrand, 'The Glivenko-Cantelli problem', Ann. of Probability 15 (1987) 837-870.

Acknowledgements Part of the work of this paper was done while the authors were visiting the M.S.R.I., Berkeley; we should like to thank the Institute for its support. The first author was partially supported by 
the Fund for Basic Research of the Israel Academy of Sciences. The second author was partially supported by grants GR/F/70730 and GR/F/31656 from the U.K. Science and Engineering Research Council. We are most grateful to M.Burke for carefully checking the manuscript.

To appear in J.S.L. 